\title{
Multifunctional Clip Strand for Regulation of DNA Strand Displacement and Construction of Complex DNA Nanodevices
}


Wang $^{1}$, Xianjin Xiao'*

${ }^{1}$ Institute of Reproductive Health and Union Hospital, Tongji Medical College, Huazhong University of Science and Technology, Wuhan, 430030, China.

${ }^{2}$ Hubei Key Laboratory of Bioinorganic Chemistry and Materia Medica, School of Chemistry and Chemical Engineering. Huazhong University of Science and Technology, Wuhan 430074, China.

*To whom correspondence should be addressed.

Email: xiaoxianjin@hust.edu.cn 


\section{Supplementary Methods.}

\section{Reaction Setup}

To a plate strip, appropriate amount of dsDNA (Probe), Invading strand (S), Clip and c-Clip solution were added. Then the plate strip was immediately put into a microplate reader (Biotek) for fluorescence measurement. The amount of dsDNA (Probe), Invading strand (S), Clip and c-Clip added in different experiments are listed in the Supplementary Table S1.

Table S1-1 Reaction setups for Clip-toehold mediated strand displacement

\begin{tabular}{lllll}
\hline \multirow{2}{*}{ Temperature } & \multicolumn{3}{l}{ Concentrations of reactants (nM) } & \\
\cline { 2 - 5 } & Probe & $\mathrm{S}$ & Clip & ThermoPol Reaction Buffer \\
\hline $37^{\circ} \mathrm{C}$ & 250 & 250 & 250 & $1 \mathrm{x}$ \\
\hline
\end{tabular}

Table S1-2 Reaction setups for Selectivity of Clip-toehold mediated strand displacement

\begin{tabular}{|c|c|c|c|c|c|c|c|}
\hline \multirow[b]{2}{*}{ Experiment } & \multirow[b]{2}{*}{ Temperature } & \multicolumn{6}{|c|}{ Concentrations of reactants (nM) } \\
\hline & & $\begin{array}{l}\text { dsDNA- } \\
1\end{array}$ & $\begin{array}{l}\text { dsDNA- } \\
2\end{array}$ & $\mathrm{~S}$ & Clip-1 & Clip-2 & $\begin{array}{l}\text { ThermoPol } \\
\text { Reaction } \\
\text { Buffer }\end{array}$ \\
\hline $0 \%$ & & 250 & 250 & 250 & - & 250 & $1 \mathrm{x}$ \\
\hline $25 \%$ & & 250 & 250 & 250 & 62.5 & 187.5 & $1 \mathrm{x}$ \\
\hline $50 \%$ & $37^{\circ} \mathrm{C}$ & 250 & 250 & 250 & 125 & 125 & $1 \mathrm{x}$ \\
\hline $75 \%$ & & 250 & 250 & 250 & 187.5 & 62.5 & $1 \mathrm{x}$ \\
\hline $100 \%$ & & 250 & 250 & 250 & 250 & - & $1 \mathrm{x}$ \\
\hline
\end{tabular}

Table S1-3 Reaction setups for Resettability of Clip-toehold mediated strand displacement Concentrations of reactants $(\mathrm{nM})$

\begin{tabular}{llllll} 
& \multicolumn{4}{l}{ Concentrations of reactants $(\mathrm{nM})$} & \\
\cline { 2 - 4 } Temperature & Probe & $\mathrm{S}$ & Clip & c-Clip & $\begin{array}{l}\text { ThermoPol } \\
\text { Reaction } \\
\text { Buffer }\end{array}$ \\
\hline $37^{\circ} \mathrm{C}$ & 250 & 250 & 250 & $262.5^{\mathrm{a}}$ & $1 \mathrm{x}$
\end{tabular}

\footnotetext{
${ }^{a}$ To make sure that the c-Clip was fully bound to the Clip strand, we set the amount of c-Clip $5 \%$ higher than that of the Clip strand.
} 
Table S1-4 Reaction setups for Allosteric mode of Clip-toehold mediated strand displacement

Concentrations of reactants $(\mathrm{nM})$

\begin{tabular}{|c|c|c|c|c|c|c|}
\hline \multirow[b]{2}{*}{ Experiment } & \multirow[b]{2}{*}{ Temperature } & \multicolumn{5}{|c|}{ Concentrations or reactants (nilvi) } \\
\hline & & Probe & $\mathrm{S}$ & $\begin{array}{l}\text { Clip- } \\
\text { Allosteric }\end{array}$ & Clip & $\begin{array}{l}\text { ThermoPol } \\
\text { Reaction } \\
\text { Buffer }\end{array}$ \\
\hline $\begin{array}{l}\text { Reaction } \\
\text { system } 1\end{array}$ & \multirow{2}{*}{$37^{\circ} \mathrm{C}$} & 250 & 250 & 250 & - & $1 \mathrm{x}$ \\
\hline $\begin{array}{l}\text { Reaction } \\
\text { system } 2\end{array}$ & & 250 & 250 & - & 250 & $1 \mathrm{x}$ \\
\hline
\end{tabular}

In the experiments of Clip-toehold based DNA walking machines, the Cargo complex and Tracks were added. The reactants of the Cargo and tracks are listed in the Supplementary Table S2.

Table S2-1 Reactants of tracks

\begin{tabular}{|c|c|c|c|c|c|c|c|}
\hline \multirow[b]{2}{*}{ Track } & \multicolumn{7}{|c|}{ Concentrations of reactants $(\mathrm{nM})$} \\
\hline & Template & S-strand & P-strand & E-strand & E-Clip & $\begin{array}{l}\text { Shared- } \\
\text { BHQ }\end{array}$ & $\begin{array}{l}\text { CutSmart }{ }^{\circledR} \\
\text { Buffer }\end{array}$ \\
\hline Track 1 & 250 & 250 & 250 & 250 & 250 & 500 & $1 \mathrm{x}$ \\
\hline Track 2 & 250 & 250 & 250 & 250 & - & 500 & $1 \mathrm{x}$ \\
\hline Track 3 & 250 & 250 & 250 & 250 & - & 250 & $1 \mathrm{x}$ \\
\hline
\end{tabular}

Table S2-2 Reactants of the Cargo complex

\begin{tabular}{lll}
\multicolumn{3}{l}{ Concentrations of reactants(nM) } \\
\hline $\begin{array}{lll}\text { walker } \\
\text { strand }\end{array}$ & $\begin{array}{l}\text { Shared- } \\
\text { FAM }\end{array}$ & Blocker \\
\hline 250 & 250 & 250 \\
\end{tabular}

In the experiments of reusable DNA logic circuits based on Clip-toehold mediated strand displacement, the concentrations of all the reactants in the reaction system were $100 \mathrm{nM}$, except for the seesaw gates and the full adder. The reactants of the seesaw gates are listed in the Supplementary Table S3-1. And the reactants of the full adder are listed in the Supplementary Table S3-2.

Table S3-1 Reactants of the seesaw gates

\begin{tabular}{lcc}
\hline \multicolumn{3}{c}{ Concentrations of reactants (nM) } \\
\hline Threshold gate & Seesaw gate & Fuel \\
\hline 20 & 100 & 200
\end{tabular}


Table S3-2 Reactants of the full adder

\begin{tabular}{|c|c|c|c|}
\hline \multicolumn{4}{|c|}{ Concentrations of reactants (nM) } \\
\hline input & Clip1-8 & AND1-4 & $\begin{array}{l}\text { Other } \\
\text { reactants }\end{array}$ \\
\hline 200 & 200 & 200 & 100 \\
\hline
\end{tabular}




\section{Supplementary Note}

a

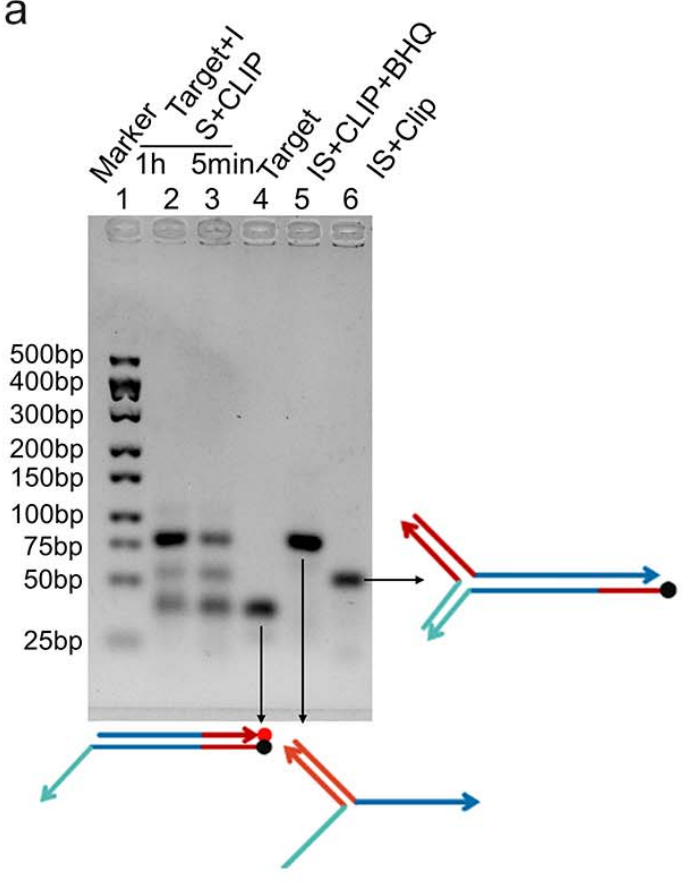

b

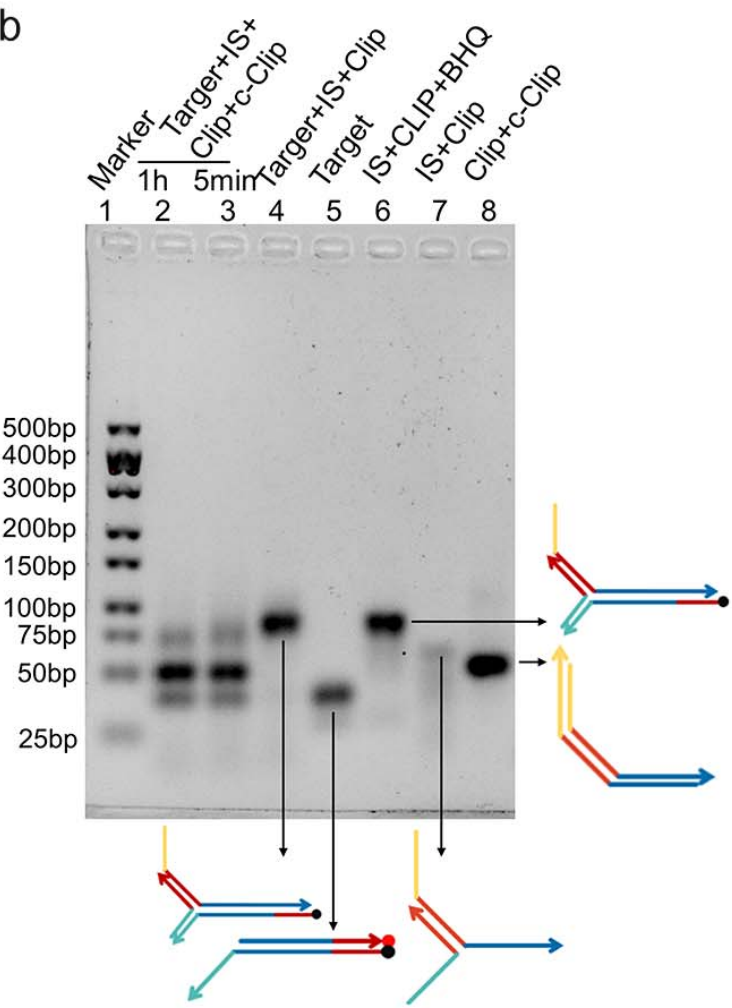

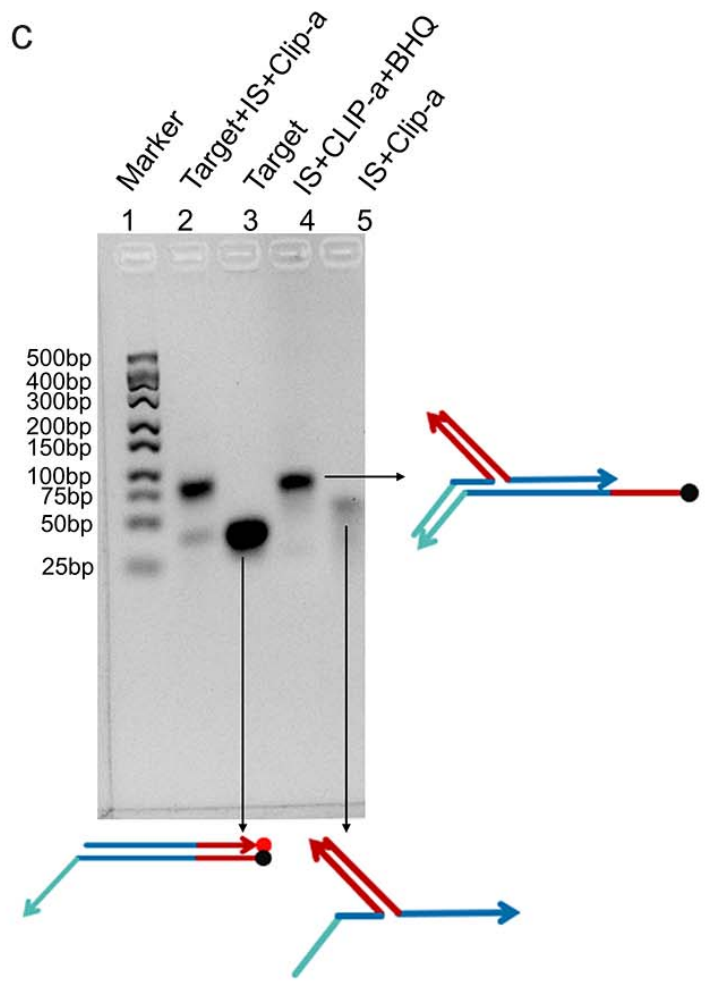

Figure S1. Agarose gel electrophoresis analysis of the Clip-toehold mediated strand displacement process. The concentration of all DNA strands was set at $10 \mathrm{uM}$. IS is the abbreviation for invading strand. (a) Verification of the Clip-toehold mediated strand displacement. (b) Verification of the allosteric Clip-toehold mediated strand displacement. (c) Verification of the resettability of the Clip-toehold mediated strand displacement. 
a



C

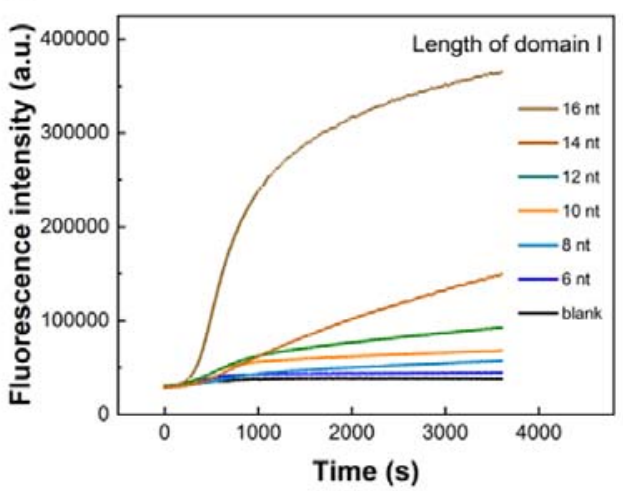

b

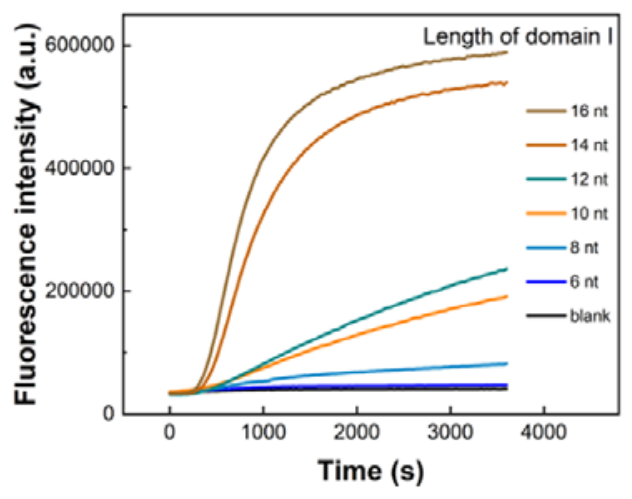

d

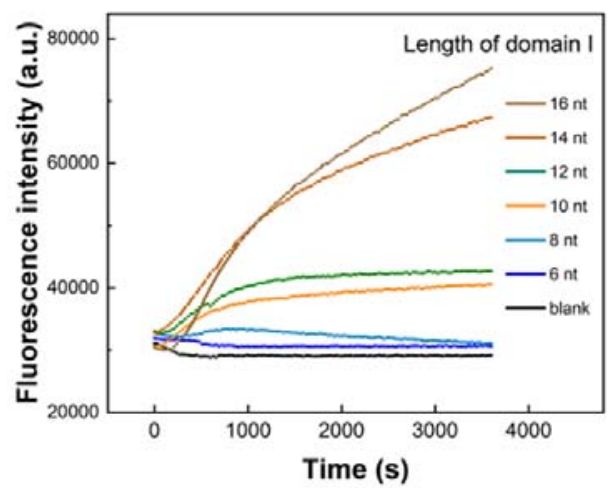

Figure S2-1. The influence of the length of domain I of the Clip on the kinetics of the strand displacement process. (a) The lengths of domain $T$ and $R$ were fixed at14 and 0 respectively. (b-d) The length of domain $T$ was fixed at 18. The length of domain R fixed at 6 (b), 12 (c) and 18 (d) Reaction settings: $250 \mathrm{nM}$ of invading strand(S4), $250 \mathrm{nM}$ of target dsDNA and $250 \mathrm{nM}$ of designated Clip. The Clips used were Clip-6-18+0+14 for (a), Clip-6-16+6+18 for (b), Clip-6-16+12+18for (c), Clip-6-16+18+18 for (d). 
a

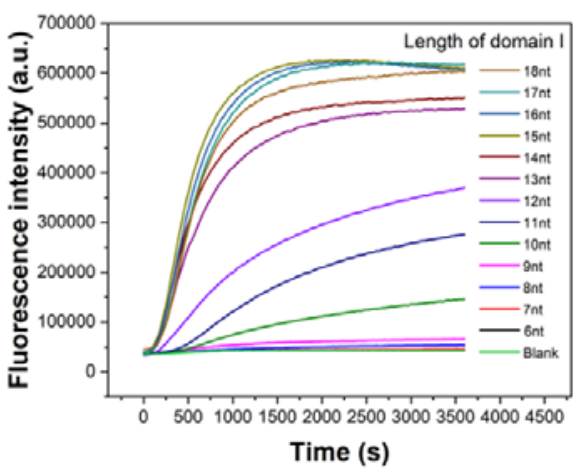

C

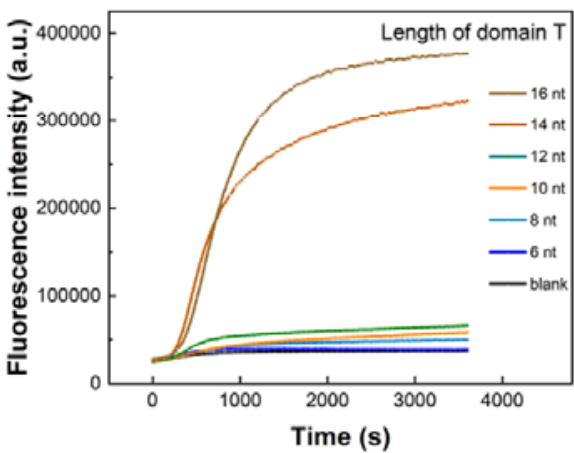

e
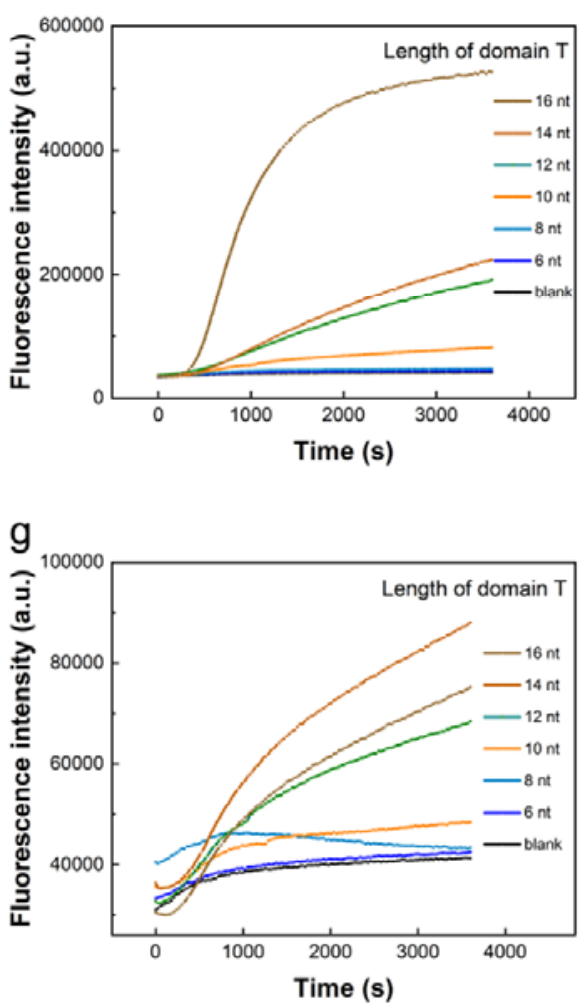

b

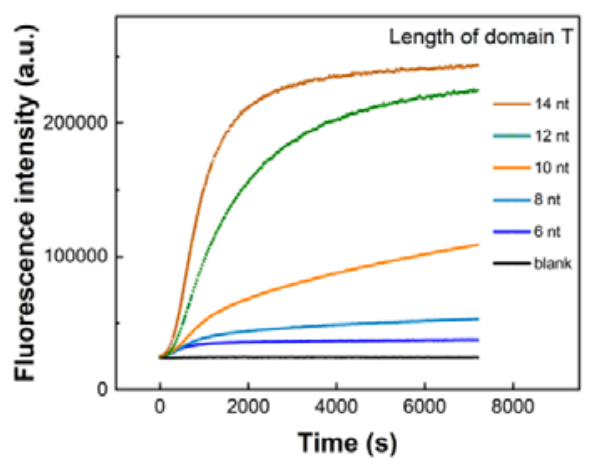

d

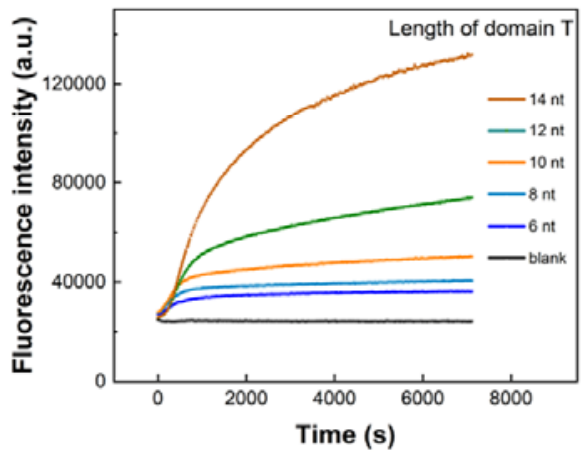

f



Figure S2-2. The influence of the length of domain T of the Clip on the kinetics of the strand displacement process. The length of domain I was fixed at 18. The length of domain R was fixed at 0 (a), 3 (b), 6 (c), 9 (d), 12 (e), 15 (f) and 18 (g). Reaction settings: $250 \mathrm{nM}$ of invading strand (S4) $250 \mathrm{nM}$ of target ds DNA and 250 nM of designated Clip. The Clips used were $18+0+6-18$ for (a),18+3+6-14 for (b) $18+6+6-16$ for (c). 18+9+614 for (d), $18+12+6-16$ for (e), $18+5+6-14$ for (f), $18+8+6-16$ for $(\mathrm{g})$. 
a

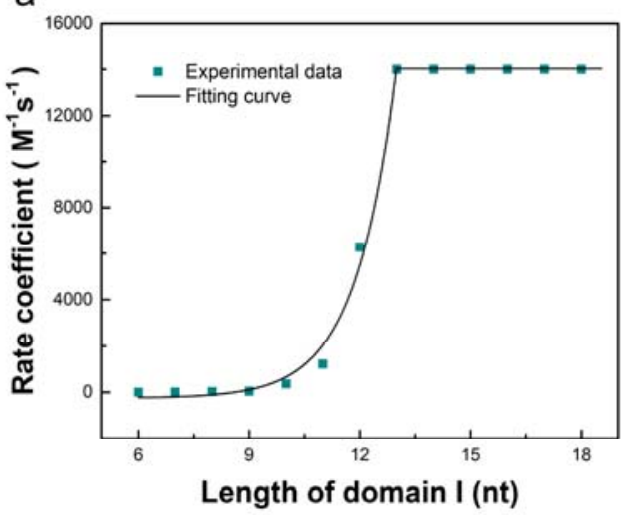

b

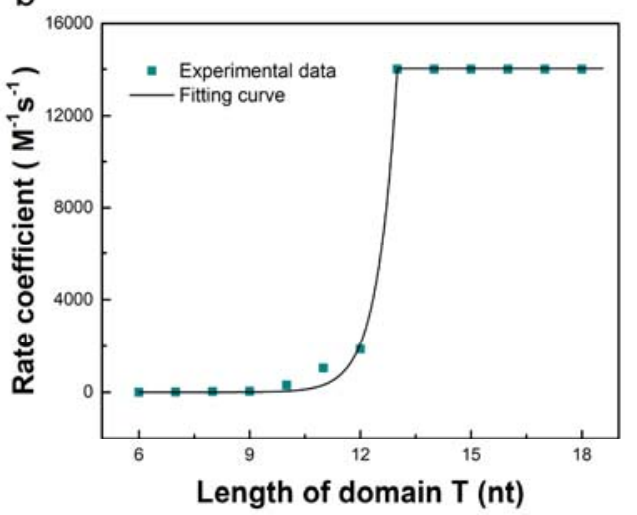

Figure S2-3 The influence of domain I and T on the kinetic of Clip-toehold mediated strand displacement. The reaction rate of Clip-toehold mediated strand displacement had an exponential positive correlation with the length of domain I (a) and T (b), which is similar to conventional toehold. 
a

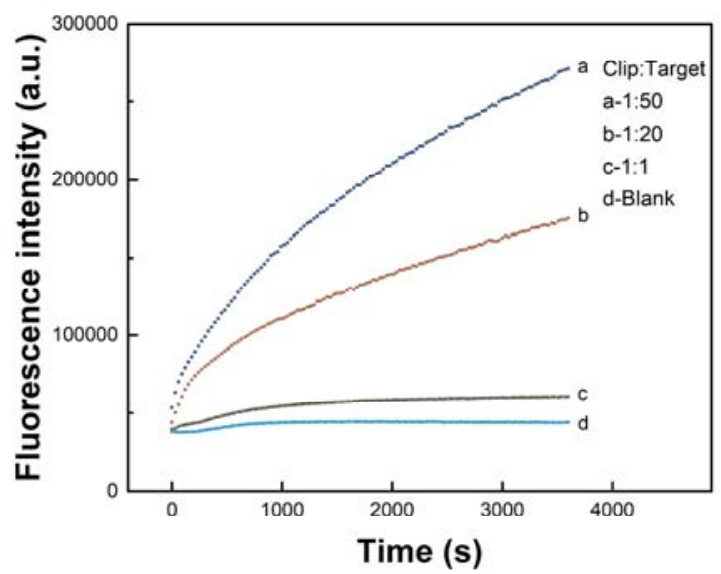

C

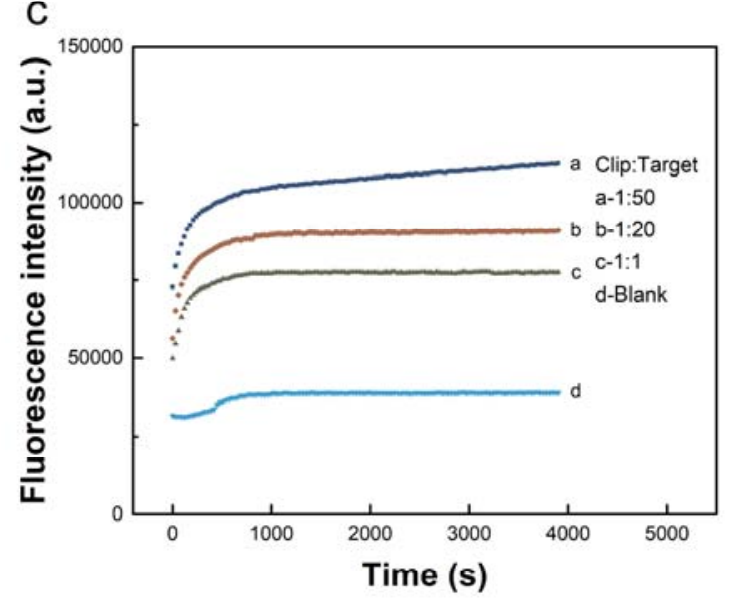

e

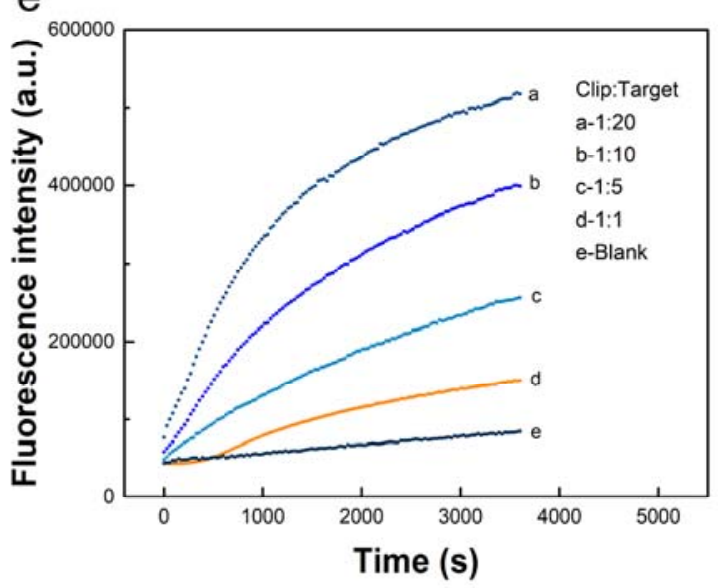

b



d

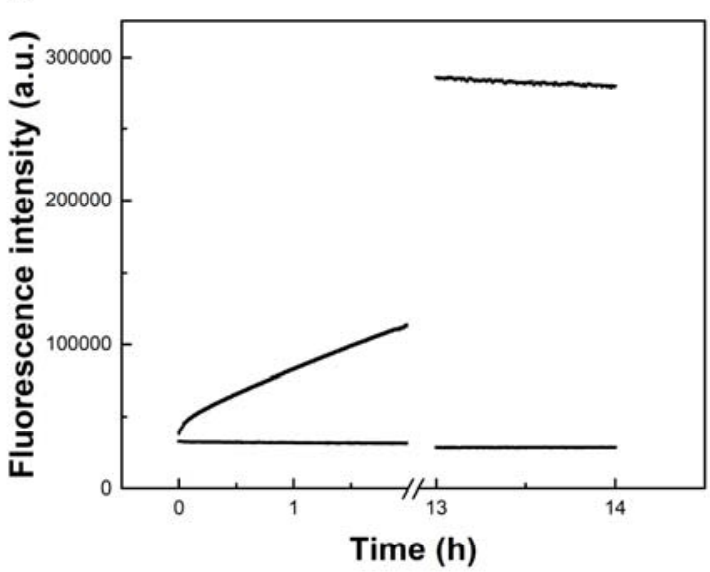

Figure S3. Investigation on the application range of Clip-toehold. The lengths of domain I, R and T were set at 8-nt, 0-nt, and 18-nt (a), 18-nt, 0-nt and 9-nt (b), 8-nt, 0-nt and 9-nt (c), 18-nt, 0-nt and 10-nt(e) respectively. The fluorescence intensity of Clip-8+0+9 in stable state (d). Reaction settings: $250 \mathrm{nM}$ of invading strand (S4), $250 \mathrm{nM}$ of target dsDNA and $250 \mathrm{nM}$ of designated amount of Clip. The Clips used were Clip-8+0+18 for (a), Clip-18+0+9 for(b), Clip-9+0+9 for (c), Clip-18+0+10 for (e). 


\section{The applicable range of Clip-toehold based on free energy change from NUPACK}

Under the sequence constraints of this experiment, the extreme condition for Clip-toehold to occur was: domain I $\geq 8$-nt and domain $T \geq 9$-nt (see the supplementary Figure 2 for details). The domain I and domain $T$ were as follows:

Domain I: GTACAGTG (I=8 nt)

Domain T: CGTTGAGTG (T=9 nt)

For Domain I : Probe-S-FAM(1), Probe-L-BHQ(2), Clip-8+0+18(3) and S(4) were simulated in NUPACK as the initial chain at $37^{\circ} \mathrm{C}$ with $50 \mathrm{mM}$ sodium ion and $0.2 \mathrm{M}$ magnesium ion.

Probe-S-FAM::Probe-L-BHQ(1::2)

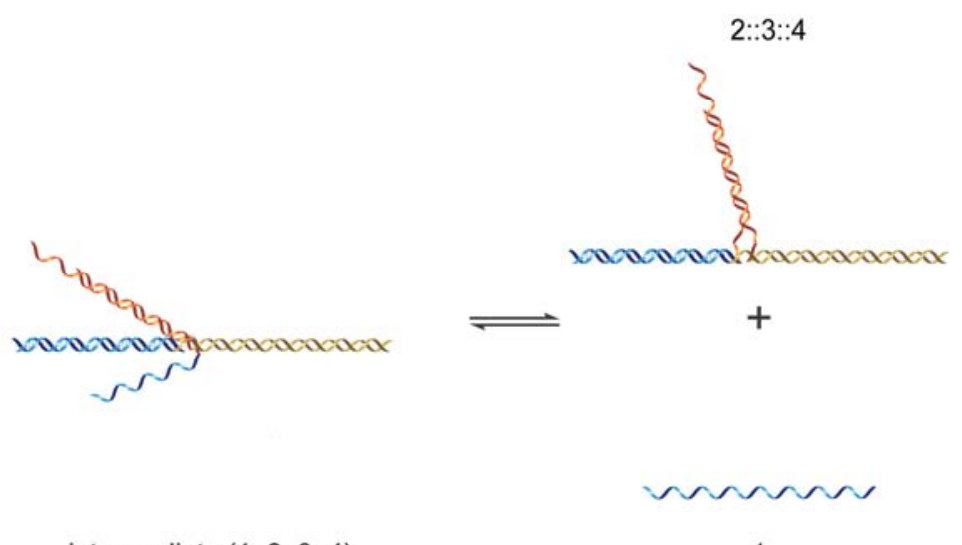

S::Clip-8+0+18(3::4)

intermediate $(1:: 2:: 3:: 4)$

1 $\Delta \mathrm{G}=-57.22 \mathrm{kcal} / \mathrm{mol}$

The free energy of the secondary structure of the intermediate $(1:: 2:: 3:: 4)$ is $-57.22 \mathrm{kcal} / \mathrm{mol}$ in NUPACK. For Domain T :Probe-S-FAM(1), Probe-L-BHQ(2), Clip-18+0+9(3) and S(4) were simulated in the NUPACK software as the initial chain at $37^{\circ} \mathrm{C}$ with $50 \mathrm{mM}$ sodium ion and $2 \mathrm{M}$ magnesium ion.

Probe-S-FAM::Probe-L-BHQ::Clip-18+0+9

$(1:: 2:: 3)$
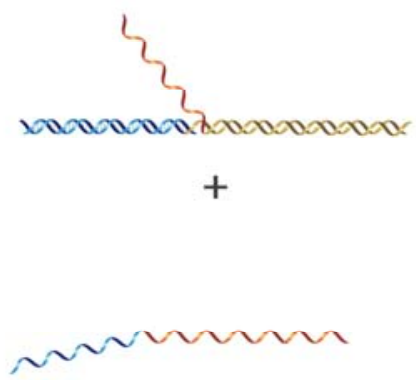

S (4)

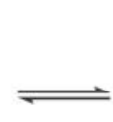

intermediate $(1:: 2:: 3:: 4)$ $\Delta \mathrm{G}=-57.41 \mathrm{kcal} / \mathrm{mol}$
$2:: 3:: 4$
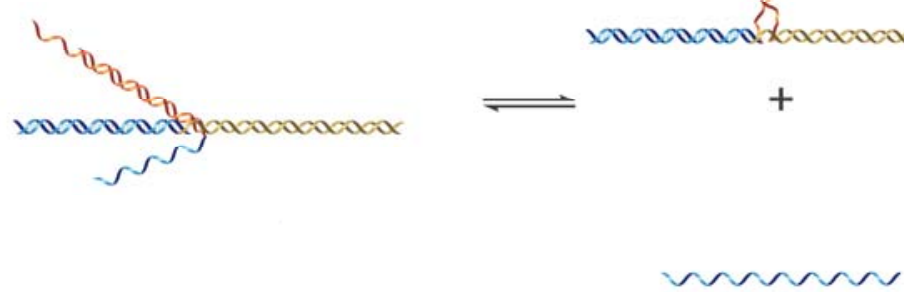

1

The free energy of the secondary structure of the intermediate $(1:: 2:: 3:: 4)$ is $-57.41 \mathrm{kcal} / \mathrm{mol}$ in NUPACK.

From the simulation results above, the free energy of the intermediate of two reaction systems are very close, which is consistent with the experimental results. Further, we could conclude that the condition for Cliptoehold to occur is: the free energy of the secondary structure of the intermediate $(1:: 2:: 3:: 4)$ is about -57 $\mathrm{kcal} / \mathrm{mol}$ or less.

Under the same conditions, if the corresponding Domain I/R changes, simulate the following groups of strands:

For Domain I: 
Group A: Probe-S-FAM, Probe-L-BHQ, Clip-6+0+18 and S. (Domain I: GGGGGG (I=6 nt)) $\Delta \mathrm{G}=-59.55 \mathrm{kcal} / \mathrm{mol}$

Group B: Probe-S-FAM, Probe-L-BHQ, Clip-5+0+18 and S. (Domain I: GGGGG (I=5 nt)) $\Delta \mathrm{G}=-57.89 \mathrm{kcal} / \mathrm{mol}$

Group C: Probe-S-FAM, Probe-L-BHQ, Clip-5+0+18 and S. (Domain I: GGGGG (I=4 nt)) $\Delta G=-56.24 \mathrm{kcal} / \mathrm{mol}$

For Domain T:

Group D: Probe-S-FAM, Probe-L-BHQ, Clip-18+0+6 and S. (Domain T: GGGGGG (I=6 nt)) $\Delta G=-57.86 \mathrm{kcal} / \mathrm{mol}$

Group E: Probe-S-FAM, Probe-L-BHQ, Clip-18+0+5 and S. (Domain T: GGGGG (I=5 nt)) $\Delta \mathrm{G}=-56.20 \mathrm{kcal} / \mathrm{mol}$

From the simulation results of group A-E, the applicable range of Clip-toehold could be expanded to 5-nt domain I and domain T. 
a



Sequence design in Fig $1 \mathrm{c}$ b



Sequence design in Fig $2 \mathrm{c}$

Figure S4-1. Schematic illustration of sequence design in Fig 1c and Fig 2c. 


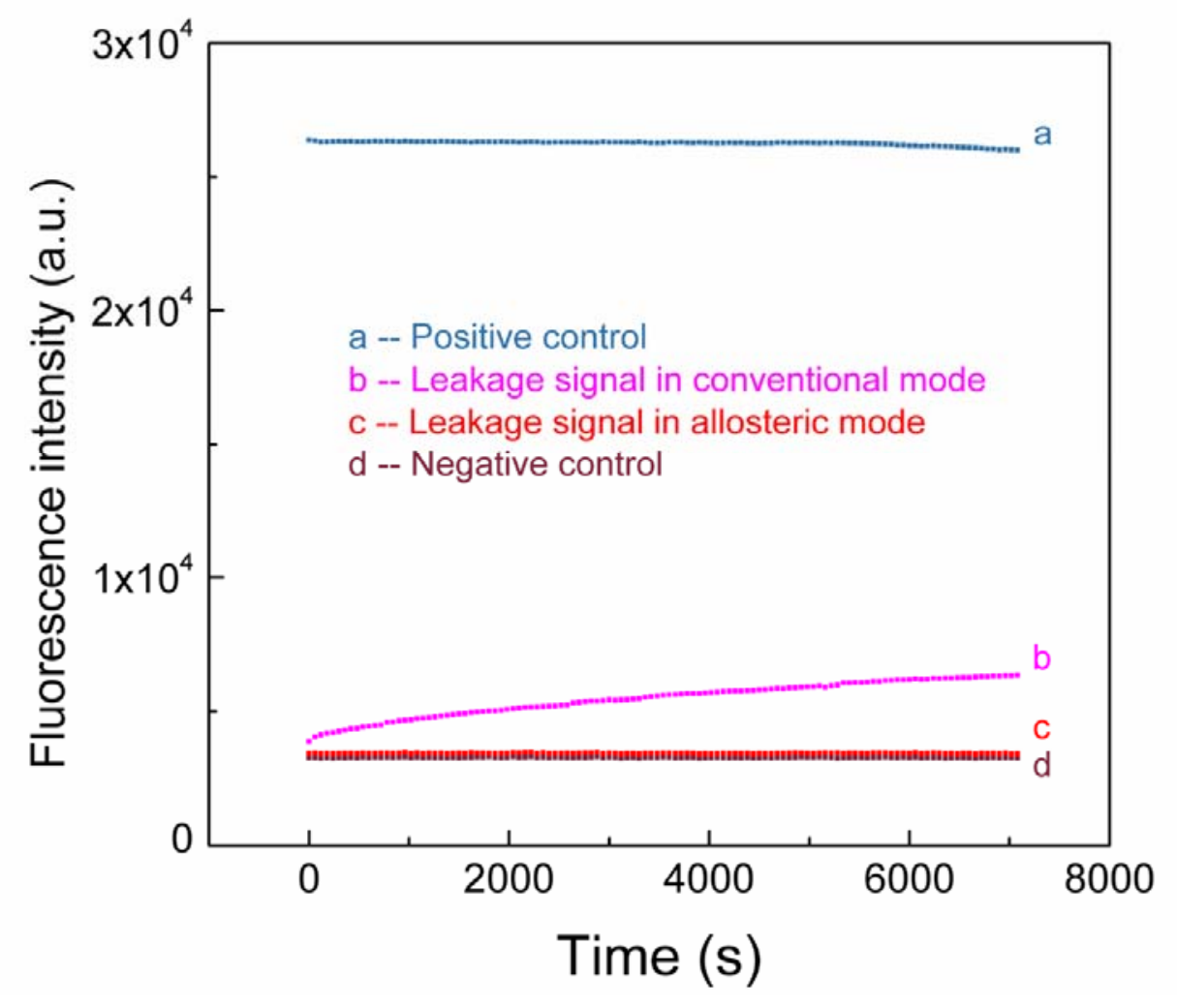

Figure S4-2. Investigation on the leakage in conventional mode and allosteric mode. In allosteric mode, the invading strand was shorter than that in conventional mode (Figure S9-1). The same amount of invading strand $(1000 \mathrm{nM})$ were added to the two systems separately. In conventional mode, leakage signal increased slowly (b). In allosteric mode, there was very few leakage signal (c). 



Figure S5. The influence of temperature on the walking process of Clip-toehold based DNA walking machine. The length of domain A of the Clip on Unit-E was set at 8-nt (a) and 3-nt (b). At $30^{\circ} \mathrm{C}$, we added the Nicking enzyme. After 9 hours of reaction, the fluorescent signals of the system reached to the maximum value. Then the signal began to decline slowly. With the reaction temperature increased from $30{ }^{\circ} \mathrm{C}$ to $37^{\circ} \mathrm{C}$, the fluorescent signal declined more rapidly. 




Figure S6. The influence of block strand on the kinetics of Clip-toehold based DNA walking machine. Reaction settings: Positive control: $250 \mathrm{nM}$ of the FAM-labelled walker strand. Negative control: $250 \mathrm{nM}$ dsDNA composed of $250 \mathrm{nM}$ of the FAM-labelled walker strand and its complementary BHQ-1-labelled strand. Reaction system: $250 \mathrm{nM}$ of the FAM-labelled walker strand and track-1 without BHQ-1-labelled strand, and 250nm of BHQ-1-labelled ssDNA. The BHQ-1-labelled ssDNA in the Reaction system could bound to the FAM-labelled walker strand. At the beginning of the reaction, the FAM-labelled walker strand was bound to track-1. 


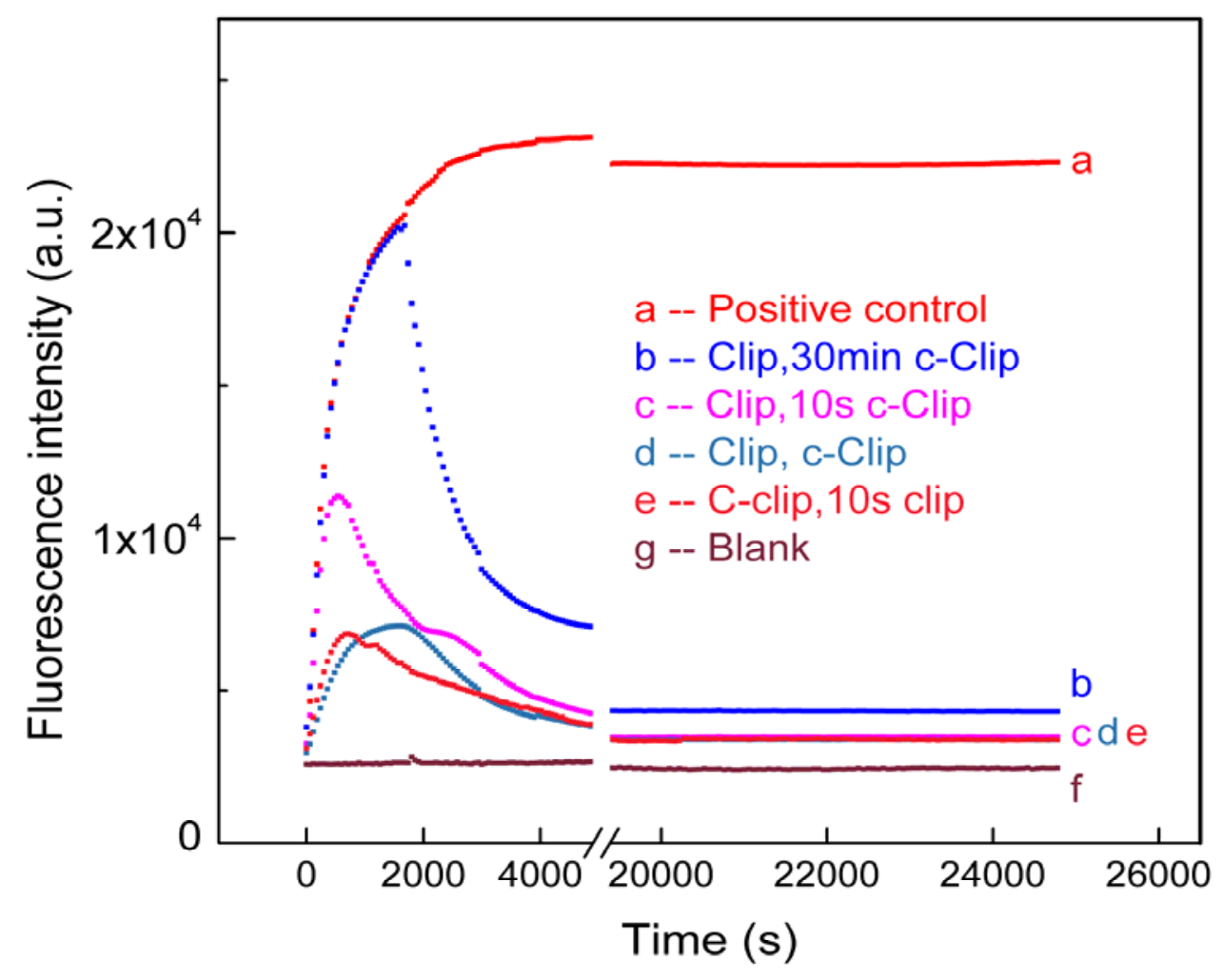

Figure S7. The influence of the time of adding Clip and c-Clip on the kinetics of Clip-toehold strand displacement reaction. (a) Positive control: after adding Clip, c-Clip was not added. (b) Clip, 30min c-Clip: after adding Clip 30min, c-Clip was added. The fluorescent signals of the reaction system began to fall after the addition of c-Clip. (c) Clip, 10s c-Clip: after adding Clip 10s, c-Clip was added. The fluorescent signal peak appeared quickly and then dropped rapidly. (d) Clip, c-Clip: Clip and c-Clip were added at the same time. The fluorescence curve in the system $d$ was similar to that in the system $c$. The fluorescent signal peak was lower in the system $d$ than that in system c. (e) c-Clip, 10s Clip: after adding c-Clip 10s, Clip was added. The fluorescence curve in the system e was similar to that in the system d. (f) Blank: neither Clip nor c-Clip was added. 

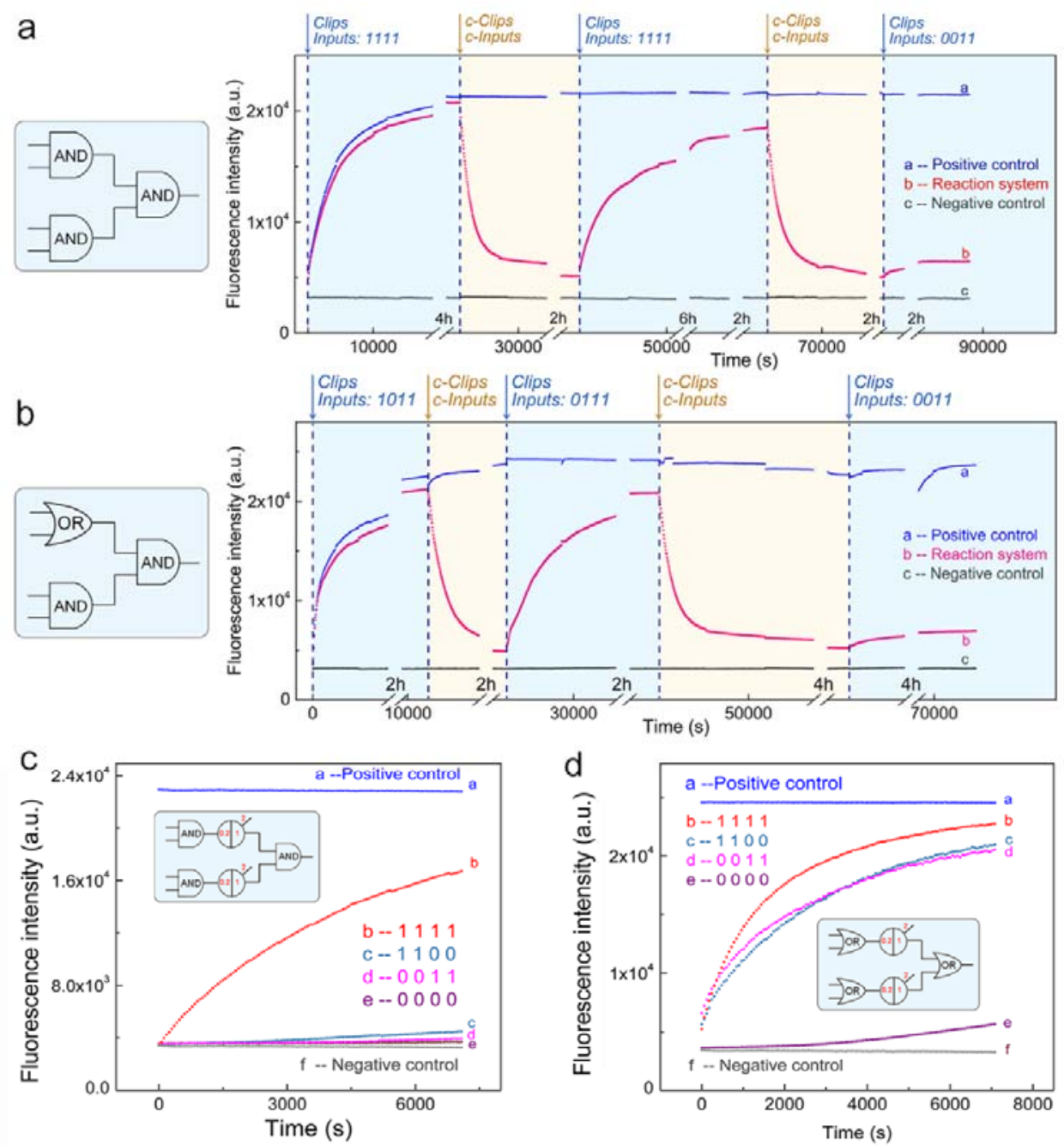

e

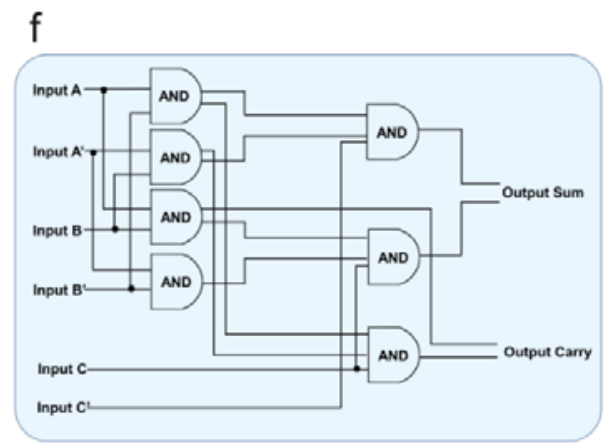

Figure S8. (a-b) Experimental verification of the reusability of two Clip-toehold based secondary DNA circuits. The reaction settings for the two experimental groups were as follows: Positive control: after adding $100 \mathrm{nM}$ of Clips and inputs at the beginning, no more reagents were added to the reaction system; Reaction system: $100 \mathrm{nM}$ of Clips and Inputs, $100 \mathrm{nM}$ of c-Clips and c-Inputs were sequentially added to the reaction system; Negative control: $100 \mathrm{nM}$ of template dsDNA (labelled with FAM and BHQ-1). (c-d) Combination of Cliptoehold based DNA circuits with the Seesaw gates. Positive control: $100 \mathrm{nM}$ of the FAM-labelled ssDNA. Negative control: $100 \mathrm{nM}$ of template dsDNA (labelled with FAM and BHQ-1). Reaction system (curve b-e): $100 \mathrm{nM}$ of Clips, Inputs and template dsDNA. (e-f) Original (e) and simplified (f) logic circuit diagram of the full adder. To simplify the design, we used fan-in strategy (Outputs of two upstream AND gates are used as input of the same downstream AND gate) to replace the OR gates. 


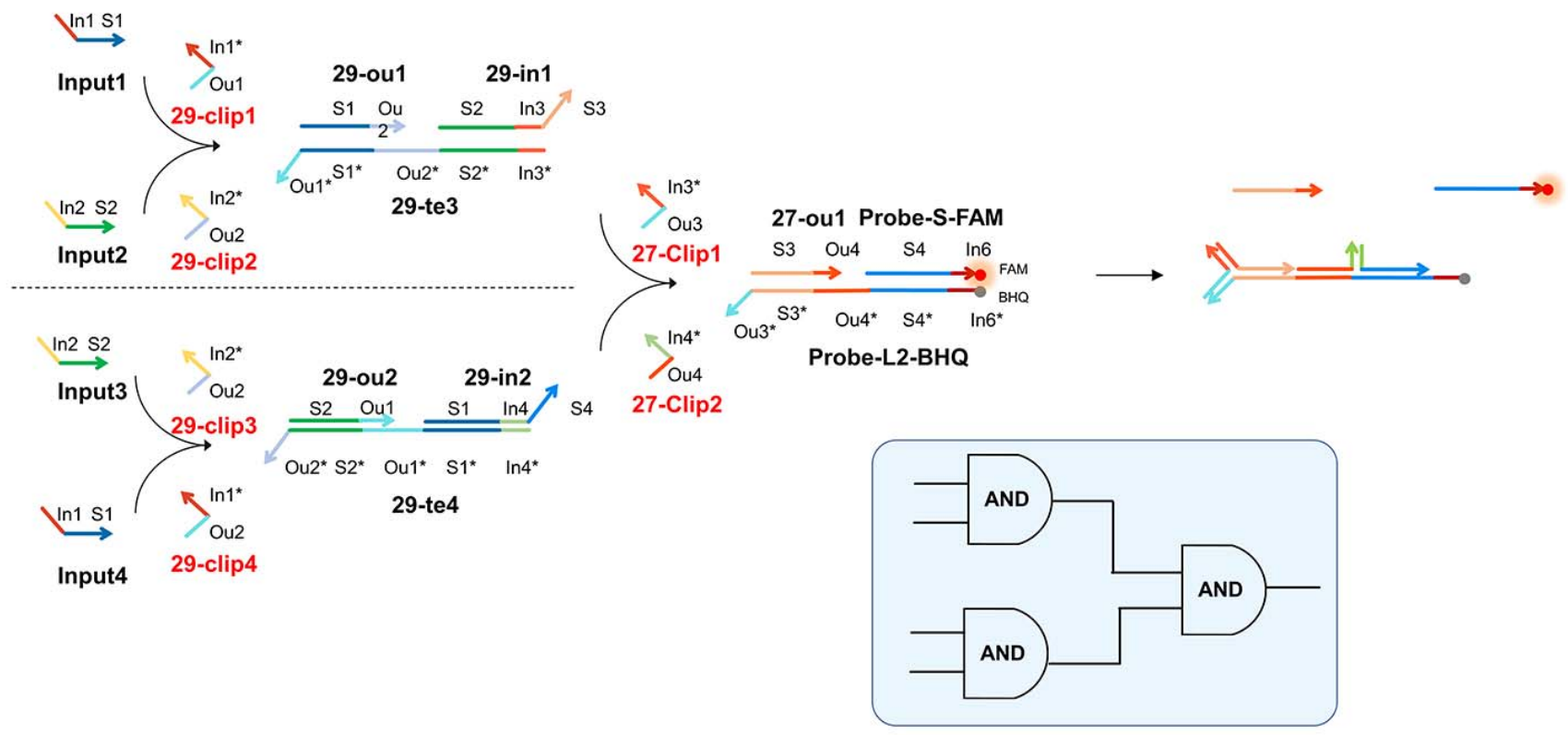

Figure S9. Schematic illustration of the secondary AND circuit. 


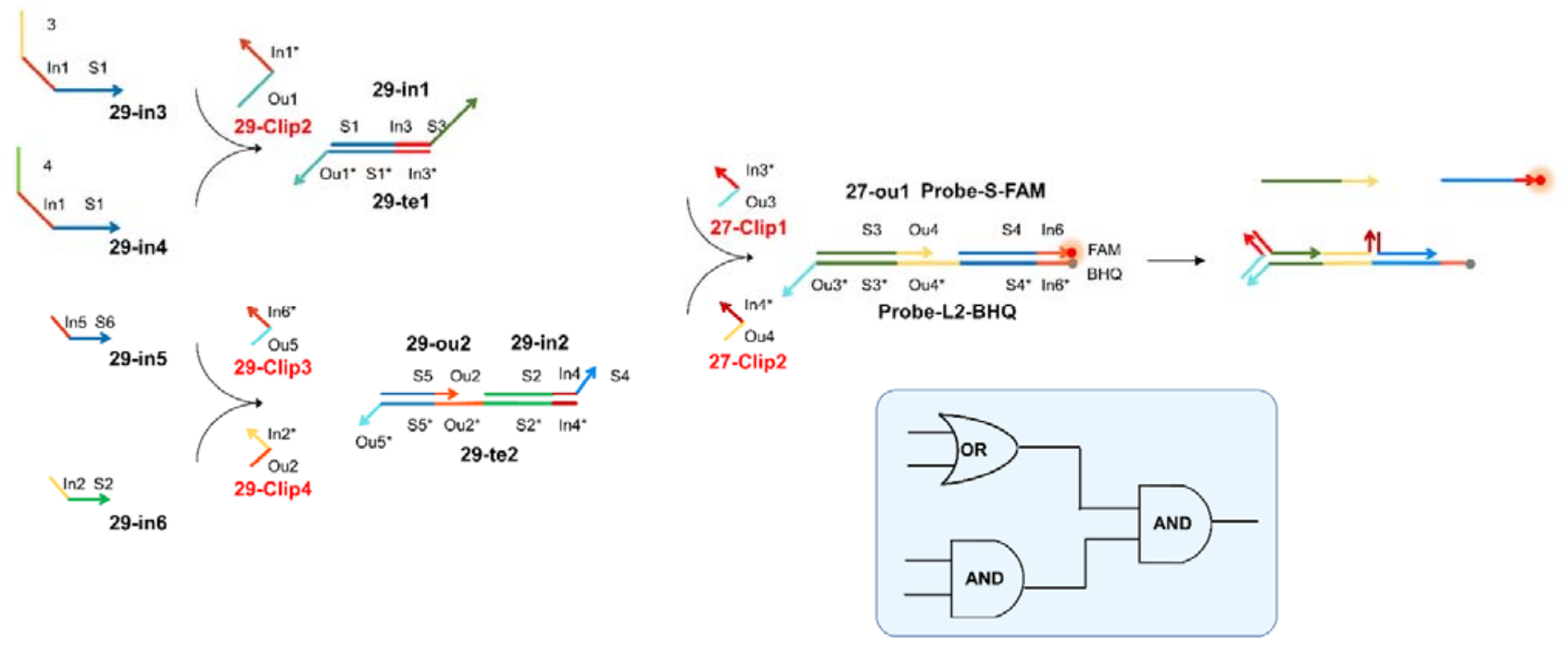

Figure S10. Schematic illustration of the Clip-toehold based secondary circuit. 


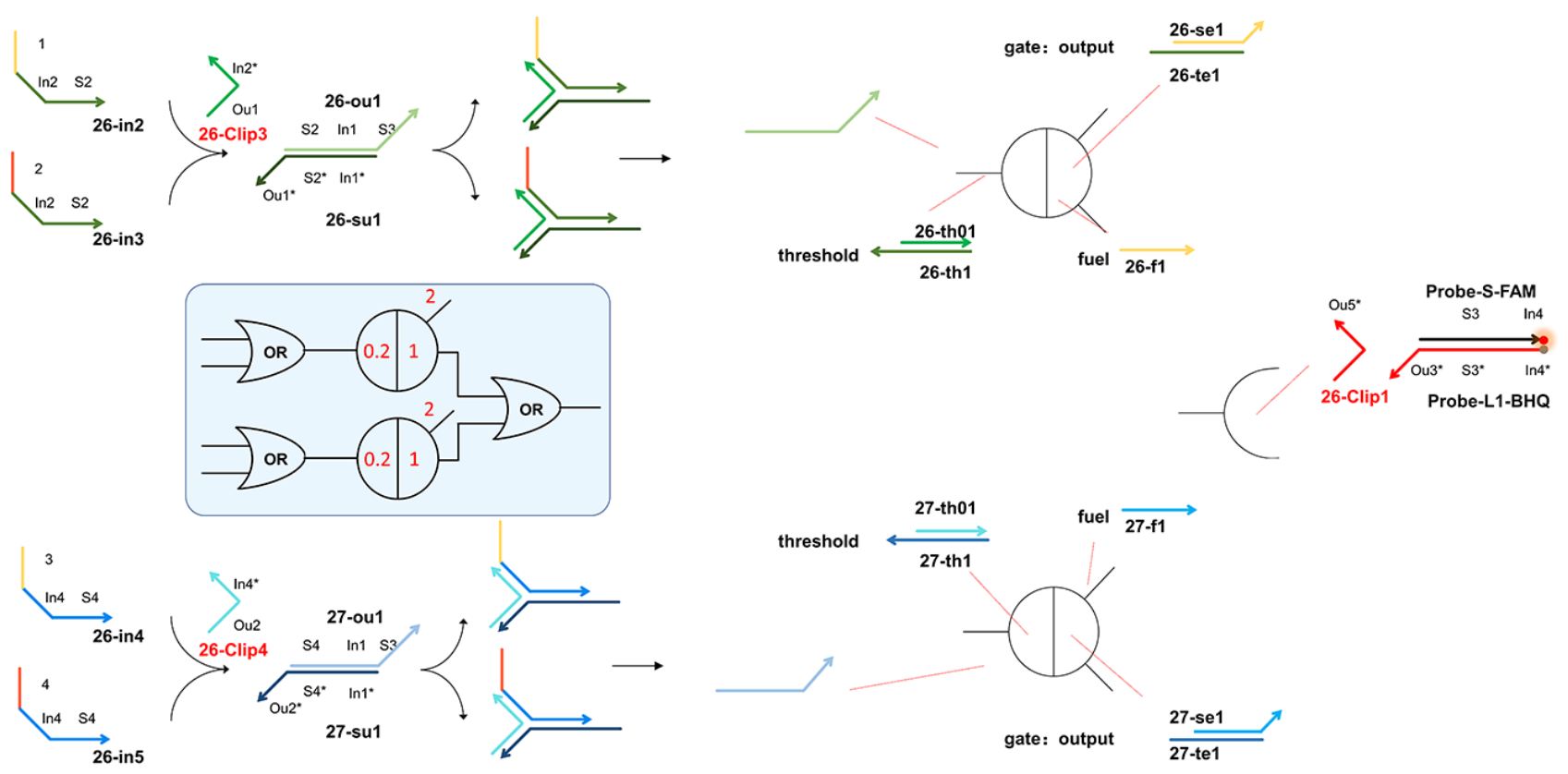

Figure S11. Schematic illustration of the combination of Clip-toehold based DNA OR circuits with the Seesaw gates. 




Figure S12. Schematic illustration of the combination of Clip-toehold based DNA AND circuits with the Seesaw gates 

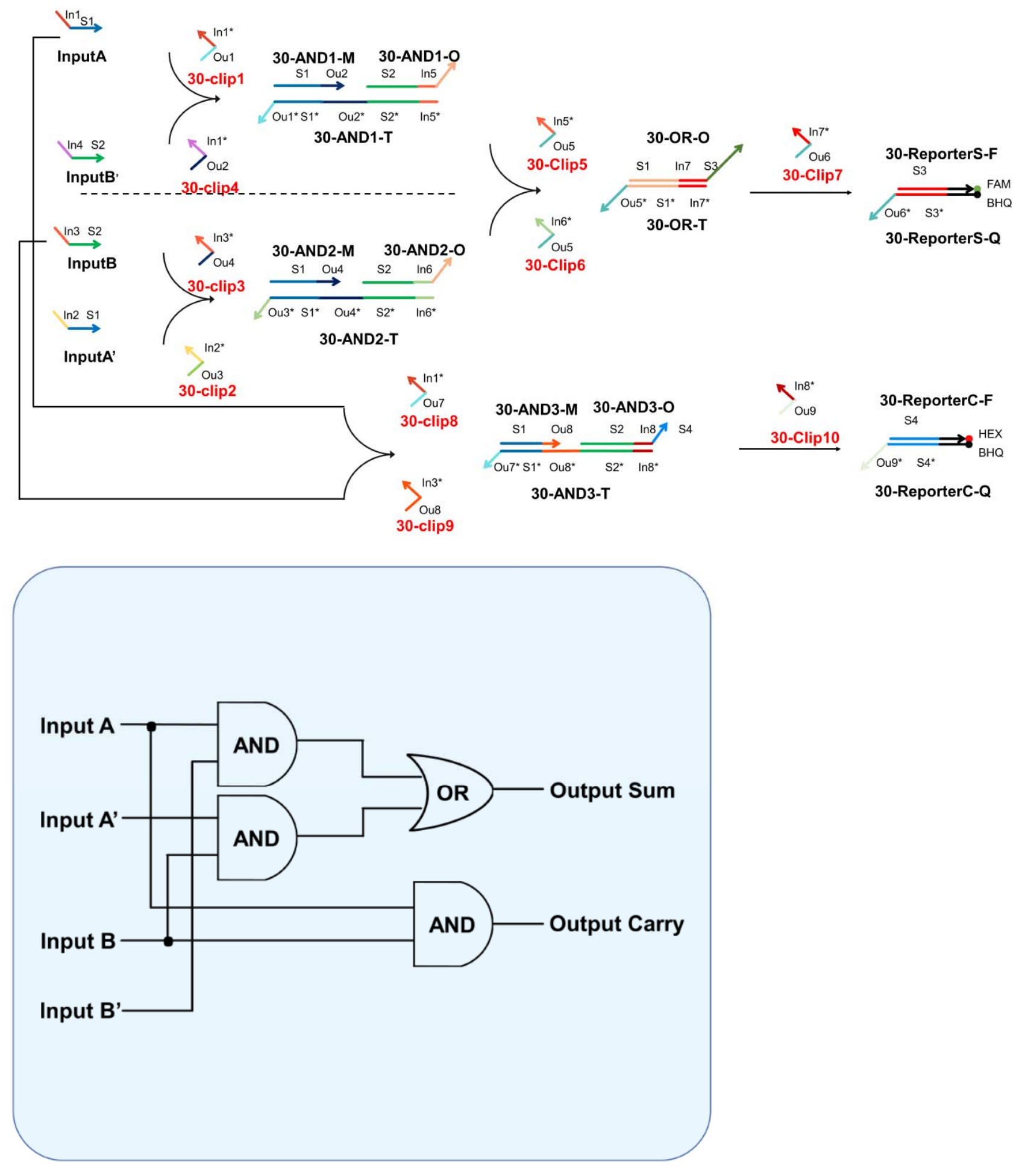

Figure S13. Schematic illustration of the half adder. 

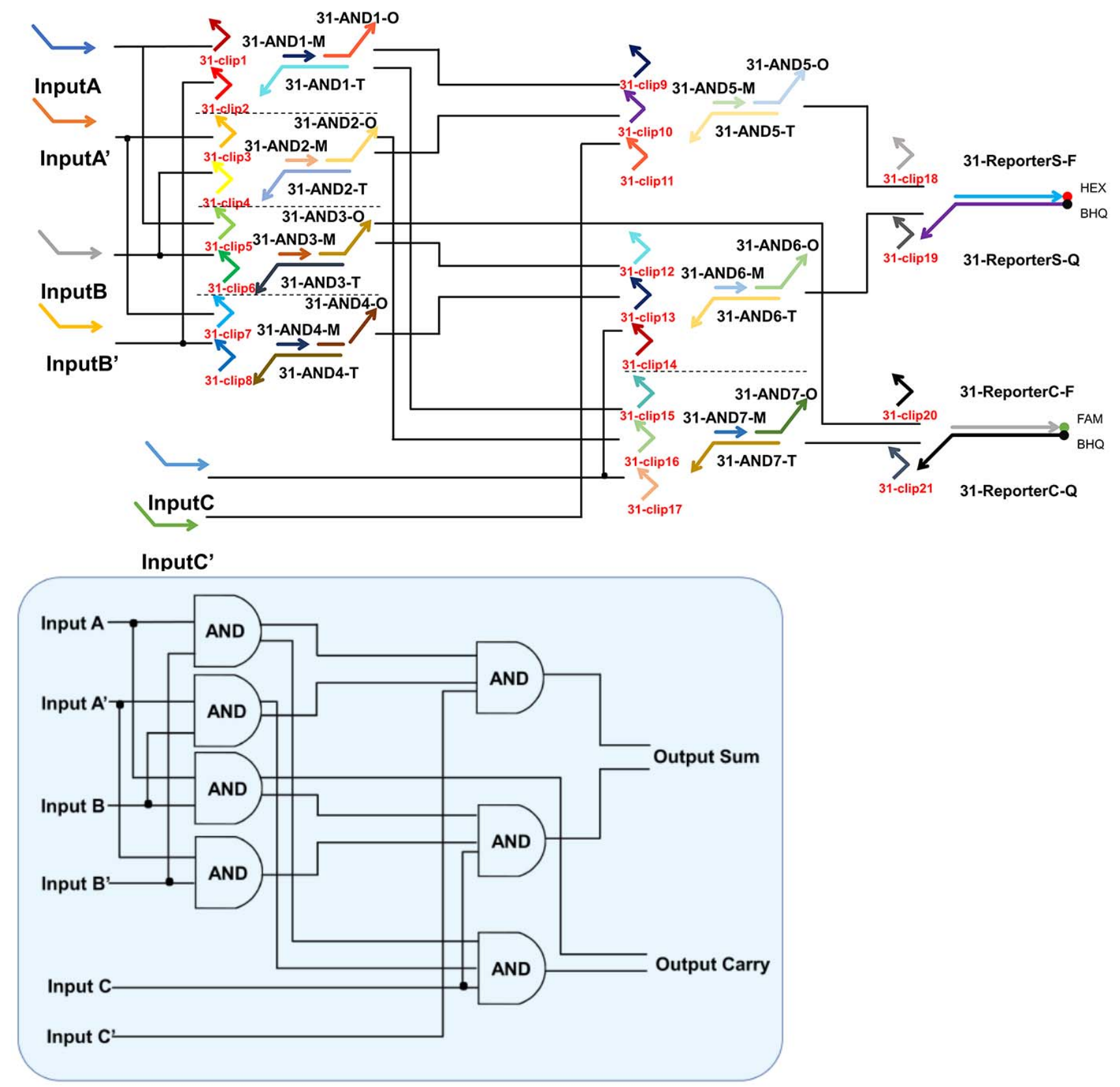

Figure S14. Schematic illustration of the full adder. 


\section{Sequences of Oligonucleotides Used}

Table S3-1 DNA sequences used in the Clip-toehold mediated strand displacement

Probe-L-BHQ2 /5`BHQ1/GTATCATAGCATAAGCAACACCCTTCCACTGTACTTCATACATG

Probe-S-FAM1 AAGGGTGTTGCTTATGCTATGATAC /3` 6-FAM/

\begin{tabular}{|c|c|}
\hline $\mathbf{S 4}$ & AGTTCACTCAACGAAGGGTGTTGCTTATGCTAT \\
\hline Clip-14+0+14 & TATGAAGTACAGTGCGTTGAGTGAACTT \\
\hline Clip-14+1+14 & TATGAAGTACAGTGACGTTGAGTGAACTT \\
\hline Clip-14+2+14 & TATGAAGTACAGTGAACGTTGAGTGAACTT \\
\hline Clip-14+3+14 & TATGAAGTACAGTGAAACGTTGAGTGAACTT \\
\hline Clip-14+4+14 & TATGAAGTACAGTGAAAACGTTGAGTGAACTT \\
\hline Clip-14+5+14 & TATGAAGTACAGTGAAAAACGTTGAGTGAACTT \\
\hline Clip-14+6+14 & TATGAAGTACAGTGAAAAAACGTTGAGTGAACTT \\
\hline Clip-14+7+14 & TATGAAGTACAGTGAAAAAAACGTTGAGTGAACTT \\
\hline Clip-14+8+14 & TATGAAGTACAGTGAAAAAAAACGTTGAGTGAACTT \\
\hline Clip-14+9+14 & TATGAAGTACAGTGAAAAAAAAACGTTGAGTGAACTT \\
\hline Clip-14+10+14 & TATGAAGTACAGTGAAAAAAAAAACGTTGAGTGAACTT \\
\hline Clip-14+11+14 & TATGAAGTACAGTGAAAAAAAAAAACGTTGAGTGAACTT \\
\hline Clip-14+12+14 & TATGAAGTACAGTGAAAAAAAAAAAACGTTGAGTGAACTT \\
\hline Clip-14+13+14 & TATGAAGTACAGTGAAAAAAAAAAAAACGTTGAGTGAACTT \\
\hline Clip-14+14+14 & TATGAAGTACAGTGAAAAAAAAAAAAAACGTTGAGTGAACTT \\
\hline Clip-14+15+14 & TATGAAGTACAGTGAAAAAAAAAAAAAAACGTTGAGTGAACTT \\
\hline Clip-14+16+14 & TATGAAGTACAGTGAAAAAAAAAAAAAAAACGTTGAGTGAACTT \\
\hline Clip-14+17+14 & TATGAAGTACAGTGAAAAAAAAAAAAAAAAACGTTGAGTGAACTT \\
\hline Clip-14+18+14 & TATGAAGTACAGTGAAAAAAAAAAAAAAAAAACGTTGAGTGAACTT \\
\hline Clip-18+0+18 & CATGTATGAAGTACAGTGCGTTGAGTGAACTTATCG \\
\hline Clip-18+1+18 & CATGTATGAAGTACAGTGACGTTGAGTGAACTTATCG \\
\hline Clip-18+2+18 & CATGTATGAAGTACAGTGAACGTTGAGTGAACTTATCG \\
\hline Clip-18+3+18 & CATGTATGAAGTACAGTGAAACGTTGAGTGAACTTATCG \\
\hline Clip-18+4+18 & CATGTATGAAGTACAGTGAAAACGTTGAGTGAACTTATCG \\
\hline Clip-18+5+18 & CATGTATGAAGTACAGTGAAAAACGTTGAGTGAACTTATCG \\
\hline Clip-18+6+18 & CATGTATGAAGTACAGTGAAAAAACGTTGAGTGAACTTATCG \\
\hline Clip-18+7+18 & CATGTATGAAGTACAGTGAAAAAAACGTTGAGTGAACTTATCG \\
\hline Clip-18+8+18 & CATGTATGAAGTACAGTGAAAAAAAACGTTGAGTGAACTTATCG \\
\hline Clip-18+9+18 & CATGTATGAAGTACAGTGAAAAAAAAACGTTGAGTGAACTTATCG \\
\hline Clip-18+10+18 & CATGTATGAAGTACAGTGAAAAAAAAAACGTTGAGTGAACTTATCG \\
\hline Clip-18+11+18 & CATGTATGAAGTACAGTGAAAAAAAAAAACGTTGAGTGAACTTATCG \\
\hline Clip-18+12+18 & CATGTATGAAGTACAGTGAAAAAAAAAAAACGTTGAGTGAACTTATCG \\
\hline Clip-18+13+18 & CATGTATGAAGTACAGTGAAAAAAAAAAAAACGTTGAGTGAACTTATCG \\
\hline Clip-18+14+18 & CATGTATGAAGTACAGTGAAAAAAAAAAAAAACGTTGAGTGAACTTATCG \\
\hline Clip-18+15+18 & CATGTATGAAGTACAGTGAAAAAAAAAAAAAAACGTTGAGTGAACTTATCG \\
\hline Clip-18+16+18 & CATGTATGAAGTACAGTGAAAAAAAAAAAAAAAACGTTGAGTGAACTTATCG \\
\hline Clip-18+17+18 & CATGTATGAAGTACAGTGAAAAAAAAAAAAAAAAACGTTGAGTGAACTTATCG \\
\hline Clip-18+18+18 & CATGTATGAAGTACAGTGAAAAAAAAAAAAAAAAAACGTTGAGTGAACTTATCG \\
\hline Clip-17+0+18 & ATGTATGAAGTACAGTGCGTTGAGTGAACTTATCG \\
\hline Clip-16+0+18 & TGTATGAAGTACAGTGCGTTGAGTGAACTTATCG \\
\hline Clip-15+0+18 & GTATGAAGTACAGTGCGTTGAGTGAACTTATCG \\
\hline Clip-14+0+18 & TATGAAGTACAGTGCGTTGAGTGAACTTATCG \\
\hline
\end{tabular}




\begin{tabular}{|c|c|}
\hline Clip-13+0+18 & ATGAAGTACAGTGCGTTGAGTGAACTTATCG \\
\hline Clip-12+0+18 & TGAAGTACAGTGCGTTGAGTGAACTTATCG \\
\hline Clip-11+0+18 & GAAGTACAGTGCGTTGAGTGAACTTATCG \\
\hline Clip-10+0+18 & AAGTACAGTGCGTTGAGTGAACTTATCG \\
\hline Clip-9+0+18 & AGTACAGTGCGTTGAGTGAACTTATCG \\
\hline Clip-8+0+18 & GTACAGTGCGTTGAGTGAACTTATCG \\
\hline Clip-7+0+18 & TACAGTGCGTTGAGTGAACTTATCG \\
\hline Clip-6+0+18 & ACAGTGCGTTGAGTGAACTTATCG \\
\hline Clip-17+6+18 & ATGTATGAAGTACAGTGAAAAAACGTTGAGTGAACTTATCG \\
\hline Clip-16+6+18 & TGTATGAAGTACAGTGAAAAAACGTTGAGTGAACTTATCG \\
\hline Clip-15+6+18 & GTATGAAGTACAGTGAAAAAACGTTGAGTGAACTTATCG \\
\hline Clip-14+6+18 & TATGAAGTACAGTGAAAAAACGTTGAGTGAACTTATCG \\
\hline Clip-13+6+18 & ATGAAGTACAGTGAAAAAACGTTGAGTGAACTTATCG \\
\hline Clip-12+6+18 & TGAAGTACAGTGAAAAAACGTTGAGTGAACTTATCG \\
\hline Clip-11+6+18 & GAAGTACAGTGAAAAAACGTTGAGTGAACTTATCG \\
\hline Clip-10+6+18 & AAGTACAGTGAAAAAACGTTGAGTGAACTTATCG \\
\hline Clip-9+6+18 & AGTACAGTGAAAAAACGTTGAGTGAACTTATCG \\
\hline Clip-8+6+18 & GTACAGTGAAAAAACGTTGAGTGAACTTATCG \\
\hline Clip-7+6+18 & TACAGTGAAAAAACGTTGAGTGAACTTATCG \\
\hline Clip-6+6+18 & ACAGTGAAAAAACGTTGAGTGAACTTATCG \\
\hline Clip-17+12+18 & ATGTATGAAGTACAGTGAAAAAAAAAAAACGTTGAGTGAACTTATCG \\
\hline Clip-16+12+18 & TGTATGAAGTACAGTGAAAAAAAAAAAACGTTGAGTGAACTTATCG \\
\hline Clip-15+12+18 & GTATGAAGTACAGTGAAAAAAAAAAAACGTTGAGTGAACTTATCG \\
\hline Clip-14+12+18 & TATGAAGTACAGTGAAAAAAAAAAAACGTTGAGTGAACTTATCG \\
\hline Clip-13+12+18 & ATGAAGTACAGTGAAAAAAAAAAAACGTTGAGTGAACTTATCG \\
\hline Clip-12+12+18 & TGAAGTACAGTGAAAAAAAAAAAACGTTGAGTGAACTTATCG \\
\hline Clip-11+12+18 & GAAGTACAGTGAAAAAAAAAAAACGTTGAGTGAACTTATCG \\
\hline Clip-10+12+18 & AAGTACAGTGAAAAAAAAAAAACGTTGAGTGAACTTATCG \\
\hline Clip-9+12+18 & AGTACAGTGAAAAAAAAAAAACGTTGAGTGAACTTATCG \\
\hline Clip-8+12+18 & GTACAGTGAAAAAAAAAAAACGTTGAGTGAACTTATCG \\
\hline Clip-7+12+18 & TACAGTGAAAAAAAAAAAACGTTGAGTGAACTTATCG \\
\hline Clip-6+12+18 & ACAGTGAAAAAAAAAAAACGTTGAGTGAACTTATCG \\
\hline Clip-17+18+18 & ATGTATGAAGTACAGTGAAAAAAAAAAAAAAAAAACGTTGAGTGAACTTATCG \\
\hline Clip-16+18+18 & TGTATGAAGTACAGTGAAAAAAAAAAAAAAAAAACGTTGAGTGAACTTATCG \\
\hline Clip-15+18+18 & GTATGAAGTACAGTGAAAAAAAAAAAAAAAAAACGTTGAGTGAACTTATCG \\
\hline Clip-14+18+18 & TATGAAGTACAGTGAAAAAAAAAAAAAAAAAACGTTGAGTGAACTTATCG \\
\hline Clip-13+18+18 & ATGAAGTACAGTGAAAAAAAAAAAAAAAAAACGTTGAGTGAACTTATCG \\
\hline Clip-12+18+18 & TGAAGTACAGTGAAAAAAAAAAAAAAAAAACGTTGAGTGAACTTATCG \\
\hline Clip-11+18+18 & GAAGTACAGTGAAAAAAAAAAAAAAAAAACGTTGAGTGAACTTATCG \\
\hline Clip-10+18+18 & AAGTACAGTGAAAAAAAAAAAAAAAAAACGTTGAGTGAACTTATCG \\
\hline Clip-9+18+18 & AGTACAGTGAAAAAAAAAAAAAAAAAACGTTGAGTGAACTTATCG \\
\hline Clip-8+18+18 & GTACAGTGAAAAAAAAAAAAAAAAAACGTTGAGTGAACTTATCG \\
\hline Clip-7+18+18 & TACAGTGAAAAAAAAAAAAAAAAAACGTTGAGTGAACTTATCG \\
\hline Clip-6+18+18 & ACAGTGAAAAAAAAAAAAAAAAAACGTTGAGTGAACTTATCG \\
\hline Clip-18+0+17 & CATGTATGAAGTACAGTGCGTTGAGTGAACTTATC \\
\hline Clip-18+0+16 & CATGTATGAAGTACAGTGCGTTGAGTGAACTTAT \\
\hline Clip-18+0+15 & CATGTATGAAGTACAGTGCGTTGAGTGAACTTA \\
\hline
\end{tabular}




\begin{tabular}{|c|c|}
\hline Clip-18+0+14 & CATGTATGAAGTACAGTGCGTTGAGTGAACTT \\
\hline Clip-18+0+13 & CATGTATGAAGTACAGTGCGTTGAGTGAACT \\
\hline Clip-18+0+12 & CATGTATGAAGTACAGTGCGTTGAGTGAAC \\
\hline Clip-18+0+11 & CATGTATGAAGTACAGTGCGTTGAGTGAA \\
\hline Clip-18+0+10 & CATGTATGAAGTACAGTGCGTTGAGTGA \\
\hline Clip-18+0+9 & CATGTATGAAGTACAGTGCGTTGAGTG \\
\hline Clip-18+0+8 & CATGTATGAAGTACAGTGCGTTGAGT \\
\hline Clip-18+0+7 & CATGTATGAAGTACAGTGCGTTGAG \\
\hline Clip-18+0+6 & CATGTATGAAGTACAGTGCGTTGA \\
\hline Clip-18+6+17 & CATGTATGAAGTACAGTGAAAAAACGTTGAGTGAACTTATC \\
\hline Clip-18+6+16 & CATGTATGAAGTACAGTGAAAAAACGTTGAGTGAACTTAT \\
\hline Clip-18+6+15 & CATGTATGAAGTACAGTGAAAAAACGTTGAGTGAACTTA \\
\hline Clip-18+6+14 & CATGTATGAAGTACAGTGAAAAAACGTTGAGTGAACTT \\
\hline Clip-18+6+13 & CATGTATGAAGTACAGTGAAAAAACGTTGAGTGAACT \\
\hline Clip-18+6+12 & CATGTATGAAGTACAGTGAAAAAACGTTGAGTGAAC \\
\hline Clip-18+6+11 & CATGTATGAAGTACAGTGAAAAAACGTTGAGTGAA \\
\hline Clip-18+6+10 & CATGTATGAAGTACAGTGAAAAAACGTTGAGTGA \\
\hline Clip-18+6+9 & CATGTATGAAGTACAGTGAAAAAACGTTGAGTG \\
\hline Clip-18+6+8 & CATGTATGAAGTACAGTGAAAAAACGTTGAGT \\
\hline Clip-18+6+7 & CATGTATGAAGTACAGTGAAAAAACGTTGAG \\
\hline Clip-18+6+6 & CATGTATGAAGTACAGTGAAAAAACGTTGA \\
\hline Clip-18+12+17 & CATGTATGAAGTACAGTGAAAAAAAAAAAACGTTGAGTGAACTTATC \\
\hline Clip-18+12+16 & CATGTATGAAGTACAGTGAAAAAAAAAAAACGTTGAGTGAACTTAT \\
\hline Clip-18+12+15 & CATGTATGAAGTACAGTGAAAAAAAAAAAACGTTGAGTGAACTTA \\
\hline Clip-18+12+14 & CATGTATGAAGTACAGTGAAAAAAAAAAAACGTTGAGTGAACTT \\
\hline Clip-18+12+13 & CATGTATGAAGTACAGTGAAAAAAAAAAAACGTTGAGTGAACT \\
\hline Clip-18+12+12 & CATGTATGAAGTACAGTGAAAAAAAAAAAACGTTGAGTGAAC \\
\hline Clip-18+12+11 & CATGTATGAAGTACAGTGAAAAAAAAAAAACGTTGAGTGAA \\
\hline Clip-18+12+10 & CATGTATGAAGTACAGTGAAAAAAAAAAAACGTTGAGTGA \\
\hline Clip-18+12+9 & CATGTATGAAGTACAGTGAAAAAAAAAAAACGTTGAGTG \\
\hline Clip-18+12+8 & CATGTATGAAGTACAGTGAAAAAAAAAAAACGTTGAGT \\
\hline Clip-18+12+7 & CATGTATGAAGTACAGTGAAAAAAAAAAAACGTTGAG \\
\hline Clip-18+12+6 & CATGTATGAAGTACAGTGAAAAAAAAAAAACGTTGA \\
\hline Clip-18+18+17 & CATGTATGAAGTACAGTGAAAAAAAAAAAAAAAAAACGTTGAGTGAACTTATC \\
\hline Clip-18+18+16 & CATGTATGAAGTACAGTGAAAAAAAAAAAAAAAAAACGTTGAGTGAACTTAT \\
\hline Clip-18+18+15 & CATGTATGAAGTACAGTGAAAAAAAAAAAAAAAAAACGTTGAGTGAACTTA \\
\hline Clip-18+18+14 & CATGTATGAAGTACAGTGAAAAAAAAAAAAAAAAAACGTTGAGTGAACTT \\
\hline Clip-18+18+13 & CATGTATGAAGTACAGTGAAAAAAAAAAAAAAAAAACGTTGAGTGAACT \\
\hline Clip-18+18+12 & CATGTATGAAGTACAGTGAAAAAAAAAAAAAAAAAACGTTGAGTGAAC \\
\hline Clip-18+18+11 & CATGTATGAAGTACAGTGAAAAAAAAAAAAAAAAAACGTTGAGTGAA \\
\hline Clip-18+18+10 & CATGTATGAAGTACAGTGAAAAAAAAAAAAAAAAAACGTTGAGTGA \\
\hline Clip-18+18+9 & CATGTATGAAGTACAGTGAAAAAAAAAAAAAAAAAACGTTGAGTG \\
\hline Clip-18+18+8 & CATGTATGAAGTACAGTGAAAAAAAAAAAAAAAAAACGTTGAGT \\
\hline Clip-18+18+7 & CATGTATGAAGTACAGTGAAAAAAAAAAAAAAAAAACGTTGAG \\
\hline Clip-18+18+6 & CATGTATGAAGTACAGTGAAAAAAAAAAAAAAAAAACGTTGA \\
\hline Clip-18+0+6 & CATGTATGAAGTACAGTGCGTTGA \\
\hline Clip-18+0+8 & CATGTATGAAGTACAGTGCGTTGAGT \\
\hline
\end{tabular}




\begin{tabular}{ll}
\hline Clip-18+0+10 & CATGTATGAAGTACAGTGGGTTGAGTGA \\
\hline Clip-18+0+12 & CATGTATGAAGTACAGTGGGTGAGTGAAC \\
\hline Clip-18+0+14 & CATGTATGAAGTACAGTGCGTTGAGTGAACTT \\
\hline Clip-18+3+6 & CATGTATGAAGTACAGTGAAACGTTGA \\
\hline Clip-18+3+8 & CATGTATGAAGTACAGTGAAAGTTGAGT \\
\hline Clip-18+3+10 & CATGTATGAAGTACAGTGAAACGTTGAGTGA \\
\hline Clip-18+3+12 & CATGTATGAAGTACAGTGAAACGTTGAGTGAAC \\
\hline Clip-18+3+14 & CATGTATGAAGTACAGTGAAACGTTGAGTGAACTT \\
\hline Clip-18+9+6 & CATGTATGAAGTACAGTGAAAAAAAAACGTTGA \\
\hline Clip-18+9+8 & CATGTATGAAGTACAGTGAAAAAAAAACGTTGAGT \\
\hline Clip-18+9+10 & CATGTATGAAGTACAGTGAAAAAAAAACGTTGAGTGA \\
\hline Clip-18+9+12 & CATGTATGAAGTACAGTGAAAAAAAAACGTTGAGTGAAC \\
\hline Clip-18+9+14 & CATGTATGAAGTACAGTGAAAAAAAAACGTTGAGTGAACTT \\
\hline Clip-18+15+6 & CATGTATGAAGTACAGTGAAAAAAAAAAAAAAACGTTGA \\
\hline Clip-18+15+8 & CATGTATGAAGTACAGTGAAAAAAAAAAAAAAACGTTGAGT \\
\hline Clip-18+15+10 & CATGTATGAAGTACAGTGAAAAAAAAAAAAAAACGTTGAGTGA \\
\hline Clip-18+15+12 & CATGTATGAAGTACAGTGAAAAAAAAAAAAAAACGTTGAGTGAAC \\
\hline Clip-18+15+14 & CATGTATGAAGTACAGTGAAAAAAAAAAAAAAACGTTGAGTGAACTT \\
\hline
\end{tabular}

Table S3-2 DNA sequences used in the experiment of Selectivity of Clip-toehold mediated strand displacement Probe-L1-BHQ /5`BHQ1/GTATCATAGCATAAGCAACACCCTTCCACTGTACTTCATACATG Probe-L1 GTATCATAGCATAAGCAACACCCTTCCACTGTACTTCATACATG

Probe-L2-BHQ /5 BHQ1/GTATCATAGCATAAGCAACACCCTTCGATTGACGAATCTACGTTAG

Probe-L2 GTATCATAGCATAAGCAACACCCTTCGATTGACGAATCTACGTTAG

Probe-S AAGGGTGTTGCTTATGCTATGATAC

S-FAM CGATAAGTTCACTCAACGAAGGGTGTTGCTTATGCTATGATAC/3`6-FAM/

Clip1-18+6+18 CATGTATGAAGTACAGTGAAAAAACGTTGAGTGAACTTATCG

Clip2-18+6+18 AACGTAGATTCGTCAATCAAAAAACGTTGAGTGAACTTATCG

Table S3-3 DNA sequences used in the experiment of Resettability of Clip-toehold mediated strand displacement

\begin{tabular}{|c|c|}
\hline Probe-L-BHQ & /5`BHQ1/GTATCATAGCATAAGCAACACCCTTCCACTGTACTTCATACATG \\
\hline Probe-S-FAM & AAGGGTGTTGCTTATGCTATGATAC /3`6-FAM/ \\
\hline $\mathbf{S}$ & CGATAAGTTCACTCAACGAAGGGTGTTGCTTATGCTAT \\
\hline Clip-18+6+18 & $\begin{array}{l}\text { GGAGGAGGAGGACATGTATGAAGTACAGTGAAAAAACGTTGAGTGAACT } \\
\text { TATCG }\end{array}$ \\
\hline c-Clip-18+6+18 & $\begin{array}{l}\text { CGATAAGTTCACTCAACGTTTTTTCACTGTACTTCATACATGTCCTCCTCCT } \\
\text { CC }\end{array}$ \\
\hline Clip-18+12+18(AC) & CATGTATGAAGTACAGTGACACACACACACCGTTGAGTGAACTTATCG \\
\hline c-Clip-18+12+18(AC) & CGATAAGTTCACTCAACGGTGTGTGTGTGTCACTGTACTTCATACATG \\
\hline
\end{tabular}

Table S3-4 DNA sequences used in the experiment of Allosteric mode of Clip-toehold mediated strand displacement

\begin{tabular}{ll} 
Probe-L-BHQ & $\mathbf{1 5} \mathbf{B H Q 1 / G T A T C A T A G C A T A A G C A A C A C C C T T C C A C T G T A C T T C A T A C A T G}$ \\
\hline Probe-S-FAM & AAGGGTGTTGCTTATGCTATGATAC /3`6-FAM/ \\
\hline $\mathbf{S}$ & AAGTTCACTCAACGGTTGCTTATGCTAT \\
\hline Clip-allosteric & GGAGGAGGAGGATGAAGTACAGTGAAGGGTAAAAAACGTTGAGTGAACTT \\
\hline
\end{tabular}




\begin{tabular}{ll}
$\mathbf{1 8}(\mathbf{6})+\mathbf{6}+\mathbf{1 4}$ & \\
\hline $\begin{array}{l}\mathbf{c - C l i p}-\mathbf{a l l o s t e r i c} \\
\mathbf{1 8}(\mathbf{6})+\mathbf{6}+\mathbf{1 4}\end{array}$ & AAGTTCACTCAACGTTTTTTACCCTTCACTGTACTTCATCCTCCTCCTCC \\
\hline $\mathbf{C l i p}-\mathbf{1 2 + 6 + 1 4}$ & GGAGGAGGAGGATGAAGTACAGTGAAAAAACGTTGAGTGAACTT \\
\hline $\mathbf{c - C l i p - 1 2 + 6 + 1 4}$ & AAGTTCACTCAACGTTTTTTCACTGTACTTCATCCTCCTCCTCC \\
\hline $\mathbf{C l i p - 1 8 + 6 + 1 4}$ & GGAGGAGGAGGACATGTATGAAGTACAGTGAAAAAACGTTGAGTGAACTT \\
\hline $\mathbf{c - C l i p - 1 8 + 6 + 1 4}$ & AAGTTCACTCAACGTTTTTTCACTGTACTTCATACATGTTCCTCCTCCTCC \\
\hline
\end{tabular}

Table S3-5 DNA sequences used in the experiment of Clip-toehold based DNA walking machines

\begin{tabular}{|c|c|}
\hline Shared-BHQ & /5'BHQ1/GGAGAGAGATAGAGGTGG \\
\hline Shared-FAM & GCGGCCTTATGCTATGATAC/3`6-FAM/ \\
\hline Template-1 & $\begin{array}{l}\text { ACTCTTGACGCACAAATACGGACGGATATAGCGCACTACCGGACAGAAAGGC } \\
\text { ATTAAAGACGG }\end{array}$ \\
\hline S-1-strand & $\begin{array}{l}\text { TCATCGAACCTCAGCCCAACTAACATCGATCAGCTACTGAACTTTTTCCGTCTT } \\
\text { TAATGCCTTTCTGT }\end{array}$ \\
\hline S-1-strand+BHQ & $\begin{array}{l}\text { CCACCTCTATCTCTCTCCTCATCGAACCTCAGCCCAACTAACATCGATCAGCTA } \\
\text { CTGAACTTTTTCCGTCTTTAATGCCTTTCTGT }\end{array}$ \\
\hline P-1-strand & TCATCGAACCTCAGCCCAACTAACATTTTTCCGGTAGTGCGCTATATCCGT \\
\hline P-1-strand+BHQ & $\begin{array}{l}\text { CCACCTCTATCTCTCTCCTCATCGAACCTCAGCCCAACTAACATTTTTCCGGTAG } \\
\text { TGCGCTATATCCGT }\end{array}$ \\
\hline E-1-strand-1 & TAGTCTAGGAGCATGAGTACTAACATTTTTCCGTATTTGTGCGTCAAGAGT \\
\hline $\begin{array}{l}\text { E-1-strand- } \\
1+\text { BHQ }\end{array}$ & $\begin{array}{l}\text { CCACCTCTATCTCTCTCCTAGTCTAGGAGCATGAGTACTAACATTTTTCCGTATT } \\
\text { TGTGCGTCAAGAGT }\end{array}$ \\
\hline E-1-strand-2 & $\begin{array}{l}\text { TAGTGTAGGAGGATGACTGCCCAACTAACATTTTTCCGTATTTGTGCGTCAAG } \\
\text { AGT }\end{array}$ \\
\hline $\begin{array}{l}\text { E-1-strand- } \\
2+B H Q\end{array}$ & $\begin{array}{l}\text { CCACCTCTATCTCTCTCCTAGTGTAGGAGGATGACTGCCCAACTAACATTTTTC } \\
\text { CGTATTTGTGCGTCAAGAGT }\end{array}$ \\
\hline E-1-strand-3 & TCATCGAACTTCAGCCCAACTAACATTTTTCCGTATTTGTGCGTCAAGAGT \\
\hline $\begin{array}{l}\text { E-1-strand- } \\
3+\text { BHO }\end{array}$ & $\begin{array}{l}\text { CCACCTCTATCTCTCTCCTCATCGAACTTCAGCCCAACTAACATTTTTCCGTATT } \\
\text { TGTGCGTCAAGAGT }\end{array}$ \\
\hline S+BHQ & TCATCGAACTTCAGCCCAACTCATGCTCCTAGACTACCCTACTTCTAG \\
\hline E-1-Clip1+BHQ & $\begin{array}{l}\text { CCACСTCTATCTCTCTCCTCATCGAACTTCAGCCCAACTCATGCTCCTAGACTA } \\
\text { CCCTACTTCTAG }\end{array}$ \\
\hline E-1-Clip2 & TCATCGAACTTCAAGTCATGCTCCTACACTACCCTACTTCTAG \\
\hline E-1-Clip2+BHQ & $\begin{array}{l}\text { CCACCTCTATCTCTCTCCTCATCGAACTTCAAGTCATGCTCCTACACTACCCTAC } \\
\text { TTCTAG }\end{array}$ \\
\hline Walker strand & $\begin{array}{l}\text { CATTCGTCTTCATGTTAGTTGGGCTGAGGTTCGATGAGTATCATAGCATAAGGC } \\
\text { CGC }\end{array}$ \\
\hline Blocker(10) & TCATCGAACTTCAGCCCAACTAACA \\
\hline Blocker(9) & CATCGAACTTCAGCCCAACTAACA \\
\hline Blocker(8) & ATCGAACTTCAGCCCAACTAACA \\
\hline Blocker(7) & TCGAACTTCAGCCCAACTAACA \\
\hline Clip-1 & AGGGTGGTAGTAGTGTTCAGTAGCTGATCGATGAAGACGAATG \\
\hline c-Clip-1 & CATTCGTCTTCATCGATCAGCTACTGAACACTACTACCACCCT \\
\hline E-1-c-Clip-1-10 & CTAGAAGTAGGGTAGTCTAGGAGCATGAGTTGGGCTGAAG \\
\hline E-1-c-Clip-1-11 & CTAGAAGTAGGGTAGTCTAGGAGCATGAGTTGGGCTGAAGT \\
\hline E-1-c-Clip-2 & CTAGAAGTAGGGTAGTCTAGGAGCATGAGTTGAAGTTCGATGA \\
\hline Template-2 & AACTGGGAAGAATCAAGTAGGAAGCAGTCAGAGAGTCAAACCAAAGATAGG \\
\hline
\end{tabular}




\begin{tabular}{|c|c|}
\hline & AACCACAGAAGG \\
\hline S-2-strand & $\begin{array}{l}\text { TCATCGAACCTCAGCCCAACTAACACTCTCTTGTCTTCGTACTTTTTTCCTTCTG } \\
\text { TGGTTCCTATCTTT }\end{array}$ \\
\hline S-2-strand+BHQ & $\begin{array}{l}\text { CCACCTCTATCTCTCTCCTCATCGAACCTCAGCCCAACTAACACTCTCTTGTCTT } \\
\text { CGTACTTTTTTCCTTCTGTGGTTCCTATCTTT }\end{array}$ \\
\hline P-2-strand & TCATCGAACCTCAGCCCAACTAACATTTTTGGTTTGACTCTCTGACTGCTT \\
\hline P-2-strand+BHQ & $\begin{array}{l}\text { CCACCTCTATCTCTCTCCTCATCGAACCTCAGCCCAACTAACATTTTTGGTTTGA } \\
\text { CTCTCTGACTGCTT }\end{array}$ \\
\hline E-2-strand-1 & TAGTCTAGGAGCATGAGTACTAACATTTTTCCTACTTGATTCTTCCCAGTT \\
\hline $\begin{array}{l}\text { E-2-strand- } \\
1+B H Q\end{array}$ & $\begin{array}{l}\text { CCACCTCTATCTCTCTCCTAGTCTAGGAGCATGAGTACTAACATTTTTCCTACTT } \\
\text { GATTCTTCCCAGTT }\end{array}$ \\
\hline E-2-strand-2 & $\begin{array}{l}\text { TACTCTACCTGCTAGTGAGCCCAACTAACATTTTTCCTACTTGATTCTTCCCAG } \\
\text { TT }\end{array}$ \\
\hline $\begin{array}{l}\text { E-2-strand- } \\
2+B H Q\end{array}$ & $\begin{array}{l}\text { CCACCTCTATCTCTCTCCTACTCTACCTGCTAGTGAGCCCAACTAACATTTTTCC } \\
\text { TACTTGATTCTTCCCAGTT }\end{array}$ \\
\hline E-2-strand-3 & TCATCGAACTTCAGCCCAACTAACATTTTTCCTACTTGATTCTTCCCAGTT \\
\hline $\begin{array}{l}\text { E-2-strand- } \\
3+\text { BHQ }\end{array}$ & $\begin{array}{l}\text { CCACCTCTATCTCTCTCCTCATCGAACTTCAGCCCAACTAACATTTTTCCTACTT } \\
\text { GATTCTTCCCAGTT }\end{array}$ \\
\hline E-2-Clip-1 & TCATCGAACTTCAGCCCAAGTCATCCTCCTACATACTTCCATCCTGAG \\
\hline E-2-Clip-1+BHQ & $\begin{array}{l}\text { CCACCTCTATCTCTCTCCTCATCGAACTTCAGCCCAAGTCATCCTCCTACATACT } \\
\text { TCCATCCTGAG }\end{array}$ \\
\hline E-2-Clip-2 & TCATCGAACTTCATCACTAGCAGGTAGAGTATTCCATCCTGAG \\
\hline E-2-Clip-2+BHQ & $\begin{array}{l}\text { CCACCTCTATCTCTCTCCTCATCGAACTTCATCACTAGCAGGTAGAGTATTCCA } \\
\text { TCCTGAG }\end{array}$ \\
\hline Clip-2 & GAAGGTATGATGCTAGTACGAAGACAAGAGAGAAGACGAATG \\
\hline c-Clip-2 & CATTCGTCTTCTCTCTCTTGTCTTCGTACTAGCATCATACCTTC \\
\hline E-2-c-Clip-1-10 & CTCAGGATGGAAGTATGTAGGAGGATGACTTGGGCTGAAG \\
\hline E-2-c-Clip-2 & CTCAGGATGGAAGTATGTAGGAGGATGACTTGAAGTTCGATGA \\
\hline Template-3 & $\begin{array}{l}\text { CCACTAAAGAACCCAAGAGCACCAAGTATGACACACTGCCAAGCGAGGGAAC } \\
\text { GTTATGAGGAA }\end{array}$ \\
\hline S-3-strand & $\begin{array}{l}\text { TCATCGAACCTCAGCCCAACTAACATGTGTCGACTGCTAGAGTTTTTTTCCTC } \\
\text { ATAACGTTCCCTCGC }\end{array}$ \\
\hline S-3-strand+BHQ & $\begin{array}{l}\text { CСАCСТCTATCTCTCTCCTCATCGAACCTCAGCCCAACTAACATGTGTCGACT } \\
\text { GCTAGAGTTTTTTTCCTCATAACGTTCCCTCGC }\end{array}$ \\
\hline P-3-strand & TCATCGAACCTCAGCCCAACTAACATTTTTTTGGCAGTGTGTCATACTTGG \\
\hline P-3-strand+BHQ & $\begin{array}{l}\text { CCACCTCTATCTCTCTCCTCATCGAACCTCAGCCCAACTAACATTTTTTTGGCAG } \\
\text { TGTGTCATACTTGG }\end{array}$ \\
\hline E-3-strand-1 & TAGTCTAGGAGCATGAGTACTAACATTTTTTGCTCTTGGGTTCTTTAGTGG \\
\hline $\begin{array}{l}\text { E-3-strand- } \\
1+B H Q\end{array}$ & $\begin{array}{l}\text { CCACCTCTATCTCTCTCCTAGTCTAGGAGCATGAGTACTAACATTTTTTGCTCTT } \\
\text { GGGTTCTTTAGTGG }\end{array}$ \\
\hline E-3-strand-2 & $\begin{array}{l}\text { TACTCTACCTGCTAGTGAGCCCAACTAACATTTTTTGCTCTTGGGTTCTTTAGT } \\
\text { GG }\end{array}$ \\
\hline $\begin{array}{l}\text { E-3-strand- } \\
2+B H Q\end{array}$ & $\begin{array}{l}\text { CCACCTCTATCTCTCTCCTACTCTACCTGCTAGTGAGCCCAACTAACATTTTTTG } \\
\text { CTCTTGGGTTCTTTAGTGG }\end{array}$ \\
\hline E-3-strand-3 & TCATCGAACTTCAGCCCAACTAACATTTTTTGCTCTTGGGTTCTTTAGTGG \\
\hline $\begin{array}{l}\text { E-3-strand- } \\
\text { 3+BHQ }\end{array}$ & $\begin{array}{l}\text { CCACCTCTATCTCTCTCCTCATCGAACTTCAGCCCAACTAACATTTTTTGCTCTT } \\
\text { GGGTTCTTTAGTGG }\end{array}$ \\
\hline E-3-Clip-1 & TCATCGAACTTCAGCCCAACTCATGCTCCTAGACTACTTGGGCTGAAG \\
\hline
\end{tabular}




\begin{tabular}{ll}
\hline $\mathbf{E - 3 - C l i p - 1 + B H Q}$ & CСACCTCTATCTCTCTCCTCATCGAACTTCAGCCCAACTCATGCTCCTAGACTA \\
& CTTGGGCTGAAG \\
\hline $\mathbf{E - 3 - C l i p - 2}$ & TCATCGAACTTCATCACTAGCAGGTAGAGTACCCTACTTCTAG \\
\hline $\mathbf{E - 3 - C l i p - 2 + B H Q}$ & CCACCTCTATCTCTCTCCTCATCGAACTTCATCACTAGCAGGTAGAGTACCCTA \\
& CTTCTAG \\
\hline $\mathbf{C l i p - 3}$ & CACAGTATCATCCACTCTAGCAGTCGACACAAAGACGAATG \\
\hline $\mathbf{c - C l i p - 3}$ & CATTCGTCTTTGTGTCGACTGCTAGAGTGGATGATACTGTG \\
\hline $\mathbf{E - 3 - c - C l i p - 1 - 1 0}$ & CTTCAGCCCAAGTAGTCTAGGAGCATGAGTTGGGCTGAAG \\
\hline $\mathbf{E - 3 - c - C l i p - 2}$ & GTAGGGTACTCTACCTGCTAGTGATGAAGTTCGATG \\
\hline
\end{tabular}

Table S3-6 DNA sequences used in the experiment of Reusable DNA logic circuits based on Cliptoehold mediated strand displacement

The primary OR circuit

\begin{tabular}{ll}
\hline Probe-L1-BHQ & /5`BHQ1/GTATCATAGCATAAGCAACACCCTTCCACTGTACTTCATACATG \\
\hline Probe-S-FAM & AAGGGTGTTGCTTATGCTATGATAC/3`6-FAM/ \\
\hline 26-in1 & AATGGATGTGAATGTTGTTGAGAGAAGGGTGTTGCTTATGCTA \\
\hline 26-in2 & AGTATGTATGAATGTTGTTGAGAGAAGGGTGTTGCTTATGCTA \\
\hline 26-Clip1 & CACCACCAATCAAGACATGTATGAAGTACAGTGCTCTCAACAACATTCA \\
\hline 26-c-Clip1 & TGAATGTTGTTGAGAGCACTGTACTTCATACATGTCTTGATTGGTGGTG \\
\hline 26-Clip2 & ACTACTCGATCAGTCCATGTATGAAGTACAGTGCTCTCAACAACATTCA \\
\hline 26-c-Clip2 & TGAATGTTGTTGAGAGCACTGTACTTCATACATGGACTGATCGAGTAGT \\
\hline
\end{tabular}

The primary AND circuit

\begin{tabular}{|c|c|}
\hline Probe-L2-BHQ & $\begin{array}{l}\text { /5`BHQ1/GTATCATAGCATAAGCAACACCCTTTGTGATTTGTATTGTGTAATG } \\
\text { TTTGTTTGATAGTGAAAAGTG }\end{array}$ \\
\hline Probe-S-FAM & AAGGGTGTTGCTTATGCTATGATAC/3`6-FAM/ \\
\hline 27-ou1 & AACAAACATTACACAATACAA \\
\hline 27-Clip1 & CACTTTTCACTATCATGGTTTTGTTATGTGTTGTATG \\
\hline 27-c-Clip1 & CATACAACACATAACAAAACCATGATAGTGAAAAGTG \\
\hline 27-in1 & CACATAACAAAACCAAACAAACCATTACACA \\
\hline 27-Clip2 & CAATACAAATCACACGTTGAGTGAACTTATAAGAGAA \\
\hline 27-c-Clip2 & TTCTCTTATAAGTTCACTCAACGTGTGATTTGTATTG \\
\hline 27-in2 & ATAAGTTCACTCAACGAAGGGTGTTGCTTATGCTA \\
\hline
\end{tabular}

The secondary AND circuit

\begin{tabular}{|c|c|}
\hline Probe-L2-BHQ & $\begin{array}{l}\text { /5`BHQ1/GTATCATAGCATAAGCAACACCCTTGTGTGTGATTTGTATTGTGTA } \\
\text { ATGTTTGTTTGATAGTGAAAAGTG }\end{array}$ \\
\hline Probe-S-FAM & AAGGGTGTTGCTTATGCTATGATAC/3`6-FAM/ \\
\hline 27-ou1 & AACAAACCATTACACAATACAA \\
\hline 27-Clip1 & CACTTTTCACTATCATGGTTTTGTTATGTGTTGTATG \\
\hline 27-c-Clip1 & CATACAACACATAACAAAACCATGATAGTGAAAAGTG \\
\hline 27-Clip2 & CAATACAAATCACACGTTGAGTGAACTTATAAGAGAA \\
\hline 27-c-Clip2 & TTCTCTTATAAGTTCACTCAACGTGTGATTTGTATTG \\
\hline 29-in1 & CATACAACATCTACACACATAACAAAACCAAACAAACCATTACACA \\
\hline 29-in2 & CATTACCAACCACCAATAAGTTCACTCAACGAAGGGTGTTGCTTATGCTA \\
\hline 29-ou1 & ATTATTCAAACCCACACAACT \\
\hline 29-ou2 & CAACTCAAACATACCACACTTC \\
\hline
\end{tabular}




\begin{tabular}{|c|c|}
\hline 29-te3 & $\begin{array}{l}\text { TATGTGTGTAGATGTTGTATGTGGTAATGAGTTGTGTGGGTTTGAATAATTGG } \\
\text { TTATAGTGTG }\end{array}$ \\
\hline 29-te4 & ACTTATTGGTGGTTGGTAATGGA GTTTGAAGTGTGGTATGTTTGAGTTGTGGA \\
\hline & TGTAATGGTG \\
\hline 29-Clip1 & CACACTATAATTCCATGATATTAGGAGTTGAATGAGT \\
\hline 29-c-Clip1 & ACTCATTCAACTCCTAATATCATGGAATTATAGTGTG \\
\hline 29-Clip2 & CACAACTCATTACCATGATGTTAGAAGATGTAGTAGA \\
\hline 29-c-Clip2 & TCTACTACATCTTCTAACATCATGGTAATGAGTTGTG \\
\hline 29-input1 & 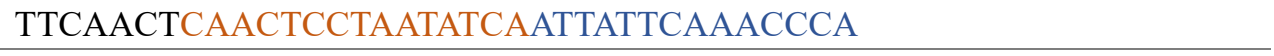 \\
\hline 29-c-input1 & TGGGTTTGAATAATTGATATTAGGAGTTGAGTTGAA \\
\hline 29-input2 & ACTAACTCATCTTCTAACATCACATACAACATCTACA \\
\hline 29-c-input2 & TGTAGATGTTGTATGTGATGTTAGAAGATGAGTTAGT \\
\hline 29-Clip3 & CACCATTACAATCCATGATTGAGTGAATTGAGATAGT \\
\hline 29-c-Clip3 & ACTATCTCAATTCACTCAATCATGGATTGTAATGGTG \\
\hline 29-Clip4 & CACACTTCAAACTCATGGAGGTAATGAATGGTTGTAT \\
\hline 29-c-Clip4 & ATACAACCATTCATTACCTCCATGAGTTTGAAGTGTG \\
\hline 29-input3 & CATCAATCAATTCACTCAATCACAACTCAAACATACC \\
\hline 29-c-input3 & GGTATGTTTGAGTTGTGATTGA GTGAATTGATTGATG \\
\hline 29-input4 & TCATTACCATTCATTACCTCCACATTACCAACCACCA \\
\hline 29-c-input4 & TGGTGGTTGGTAATGTGGAGGTAATGAATGGTAATGA \\
\hline
\end{tabular}

The secondary AND\&OR circuit

\begin{tabular}{|c|c|}
\hline Probe-L2-BHQ & $\begin{array}{l}\text { /5`BHQ1/GTATCATAGCATAAGCAACACCCTTGTGTGTGATTTGTATTGTGTA } \\
\text { ATGTTTGTTTGATAGTGAAAAGTG }\end{array}$ \\
\hline Probe-S-FAM & AAGGGTGTTGCTTATGCTATGATAC/3`6-FAM/ \\
\hline 27-ou1 & AACAAACCATTACACAATACAA \\
\hline 27-Clip1 & CACTTTTCACTATCATGGTTTTGTTATGTGTTGTATG \\
\hline 27-c-Clip1 & CATACAACACATAACAAAACCATGATAGTGAAAAGTG \\
\hline 27-Clip2 & CAATACAAATCACACGTTGAGTGAACTTATAAGAGAA \\
\hline 27-c-Clip2 & TTCTCTTATAAGTTCACTCAACGTGTGATTTGTATTG \\
\hline 29-in2 & CATTACCAACCACCAATAAGTTCACTCAACGAAGGGTGTTGCTTATGCTA \\
\hline 29-ou2 & CAACTCAAACATACCACACTTC \\
\hline 29-te2 & $\begin{array}{l}\text { ACTTATTGGTGGTTGGTAATGTGAGTTTGAAGTGTGGTATGTTTGAGTTGTGC } \\
\text { TTGTAATGGTG }\end{array}$ \\
\hline 29-Clip3 & CACCATTACAATCCATGATTGAGTGAATTGAGATAGT \\
\hline 29-c-Clip3 & ACTATCTCAATTCACTCAATCATGGATTGTAATGGTG \\
\hline 29-Clip4 & CACACTTCAAACTCATGGAGGTAATGAATGGTTGTAT \\
\hline 29-c-Clip4 & ATACAACCATTCATTACCTCCATGAGTTTGAAGTGTG \\
\hline 29-in5 & CATCAATCAATTCACTCAATCACAACTCAAACATACC \\
\hline 29-c-in5 & GGTATGTTTGAGTTGTGATTGAGTGAATTGATTGATG \\
\hline 29-in6 & TCATTACCATTCATTACCTCCACATTACCAACCACCA \\
\hline 29-c-in6 & TGGTGGTTGGTAATGTGGAGGTAATGAATG GTAATGA \\
\hline 29-in1 & CATACAACATCTACACACATAACAAAACCAA ACAAACCATTACACA \\
\hline 29-te1 & TATGTGTGTAGATGTTGTATGTGGTTATGGATATTG \\
\hline 29-Clip2 & CAATATCCATAACCATGATGTTAGAAGATGTAGTAGA \\
\hline 29-c-Clip2 & TCTACTACATCTTCTAACATCATGGTTATGGATATTG \\
\hline 29-in3 & ACTAACTCATCTTCTAACATCACATACAACATCTACA \\
\hline
\end{tabular}




\begin{tabular}{|c|c|}
\hline 29-c-in3 & TGTAGATGTTGTATGTGATGTTAGAAGATGAGTTAGT \\
\hline 29-in4 & ATCAATCCATCTTCTAACATCACATACAACATCTACA \\
\hline 29-c-in4 & TGTAGATGTTGTATGTGATGTTAGAAGATGGATTGAT \\
\hline \multicolumn{2}{|c|}{ Combination of Clip-toehold based DNA OR circuits with the Seesaw gates } \\
\hline Probe-L1-BHQ & /5'BHQ1/GTATCATAGCATAAGCAACACCCTTCCACTGTACTTCATACATG \\
\hline Probe-S-FAM & AAGGGTGTTGCTTATGCTATGATAC/3`6-FAM/ \\
\hline \multirow[t]{2}{*}{ 26-Clip2 } & CACCACCAATCAAGA \\
\hline & CATGTATGAAGTACAGTGTTGAGTGAACTTAT \\
\hline 26-c-Clip2 & ATAAGTTCACTCAACACTGTACTTCATACATGTCTTGATTGGTGGTG \\
\hline \multirow[t]{2}{*}{ 26-se1 } & CATATCTAATCTCCA \\
\hline & ATAAGTTCACTCAACGAAGGGTGTTGCTTATGCTA \\
\hline 26-te1 & TGAACTTATTGGAGATTAGATATGAGAGTAA \\
\hline 26-f1 & CATATCTAATCTCCAATAAGTTCA \\
\hline 26-th1 & TGGAGATTAGATATGAGAGTAATGGTG \\
\hline 26-th01 & CATATCTAATCTCCA \\
\hline 26-ou1 & CATTACCAACCACCATTACTCTCATATCTAATCTCCA \\
\hline 26-su1 & AGAGTAATGGTGGTTGGTAATGATGAGGTGGGAGAATG \\
\hline 26-Clip3 & CATTCTCССАССТСАТСААААСТСТСТСТСАТАТТСАТС \\
\hline 26-c-Clip3 & GATGAATATGAGAGAGAGTTTTGATGAGGTGGGAGAATG \\
\hline 26-in2 & ATGAGAGAGAGTTTTGCATTACCAACCACCAACTACTA \\
\hline 26-in3 & ATGAGAGAGAGTTTTGCATTACCAACCACCAATAACAC \\
\hline 27-se1 & CAATCTAACACTCCAATAAGTTCACTCAACGAAGGGTGTTGCTTATGCTA \\
\hline 27-te1 & TGAACTTATTGGAGTGTTAGATTGAGTAGAT \\
\hline 27-f1 & CAATCTAACACTCCAATAAGTTCA \\
\hline 27-th1 & TGGAGTGTTAGATTGAGTAGATTGATT \\
\hline 27-th01 & CAATCTAACACTCCA \\
\hline 27-ou1 & CAATTCACTCAATCAATCTACTCAATCTAACACTCCAAT \\
\hline 27-su1 & TGAACTTATTGGAGTGTTAGATTGTGGAGGTAATGAATG \\
\hline 26-Clip4 & CATTCATTACCTCCACATCCTATTCAAACAATCACTA \\
\hline 26-c-Clip4 & TAGTGATTGTGAATTGTAGATGTGGAGGTAATGAATG \\
\hline 26-in4 & TGTTTGAATAGGATCAATTCACTCAATCAACTACT \\
\hline 26-in5 & TGTTTGAATAGGATCAATTCACTCAATCAATATCAC \\
\hline
\end{tabular}

Combination of Clip-toehold based DNA AND circuits with the Seesaw gates

Probe-L2-BHQ /5 BHQ1/GTATCATAGCATAAGCAACACCCTTGTGTGATTTGTATTGTGTAAT GTTTGTTTGATAGTGAAAAGTG

\begin{tabular}{|c|c|}
\hline Probe-S-FAM & AAGGGTGTTGCTTATGCTATGATAC/3`6-FAM/ \\
\hline 27-ou1 & AACAAACCATTACACAATACAA \\
\hline 27-Clip1 & CACTTTTCACTATCATGGTTTTGTTATGTGTTGTATG \\
\hline 27-c-Clip1 & CATACAACACATAACAAAACCATGATAGTGAAAAGTG \\
\hline 27-Clip2 & CAATACAAATCACACGTTGAGTGAACTTATAAGAGAA \\
\hline 27-c-Clip2 & TTCTCTTATAAGTTCACTCAACGTGTGATTTGTATTG \\
\hline \multirow[t]{2}{*}{ 26-se1 } & CATATCTAATCTCCA \\
\hline & ATAAGTTCACTCAACGAAGGGTGTTGCTTATGCTA \\
\hline 26-te1 & TGAACTTATTGGAGATTAGATATGAGAGTAA \\
\hline 26-f1 & CATATCTAATCTCCAATAAGTTCA \\
\hline
\end{tabular}




\begin{tabular}{|c|c|}
\hline 26-th1 & TGGAGATTAGATATGAGAGTAATGG \\
\hline 26-th01 & CATATCTAATCTCCA \\
\hline 26-ou1 & CATTACCAACCACCATTACTCTCATATCTAATCTCCA \\
\hline 27-ou2-t & $\begin{array}{l}\text { AGAGTAATGGTGGTTGGTAATGTGAGTTTGAAGTGTGGTATGTTTGAGTTGT( } \\
\text { ATTGTAATGGTG }\end{array}$ \\
\hline 27-ou2 & CAACTCAAACATACCACACTTC \\
\hline 27-Clip3 & CACCATTACAATCCATGATTGAGTGAATTGAGATAGT \\
\hline 27-c-Clip3 & ACTATCTCAATTCACTCAATCATGGATTGTAATGGTG \\
\hline 27-in2 & CAATTCACTCAATCACAACTCAAACATACC \\
\hline 27-Clip4 & CACACTTCAAACTCATGGAGGTAATGAATGGTTGTAT \\
\hline 27-c-Clip4 & ATACAACCATTCATTACCTCCATGAGTTTGAAGTGTG \\
\hline 27-in3 & CATTCATTACCTCCACATTACCAACCACCA \\
\hline 28-se1 & CAATCTAACACTCCACACATAACAAAACCAAACAAACCATTACACA \\
\hline 28-te1 & GTTATGTGTGGAGTGTTAGATTGAGTAGAT \\
\hline 28-f1 & CAATCTAACACTCCACACATAAC \\
\hline 28-th1 & TGGAGTGTTAGATTGAGTAGATTGATT \\
\hline 28-th01 & CAATCTAACACTCCA \\
\hline 27-ou1 & CAATTCACTCAATCAATCTACTCAATCTAACACTCCACA \\
\hline 27-ou3-t & $\begin{array}{l}\text { TGAACTTATTGGAGTGTTAGATTGTGGTAATGAGTTGTGTGGGTTTGAATAA } \\
\text { TTGGAATTATAGTGTG }\end{array}$ \\
\hline 27-ou3 & ATTATTCAAACCCACACAACT \\
\hline 27-Clip5 & CACACTATAATTCCATGATATTAGGAGTTGAATGAGT \\
\hline 27-in4 & TTCAACTCAACTCCTAATATCAATTATTCAAACCCA \\
\hline 27-Clip6 & CACAACTCATTACCATGATGTTAGAAGATGTAGTAGA \\
\hline 27-in5 & ACTAACTCATCTTCTAACATCACAATTCACTCAATCA \\
\hline
\end{tabular}

Half adder

\begin{tabular}{|c|c|}
\hline 30-ReporterS-Q & /5'BHQ1/ATAGCATAAGCAACACCCTTCACTGTACTTCATA \\
\hline 30- ReporterS-F & AAGGGTGTTGCTTATGCTAT/3`6-FAM/ \\
\hline 30- ReporterC-Q & /5 BHQ1/ATGAATTGGGTGGGAGGTGGTGA GGTGTGGAGAGGA \\
\hline 30- ReporterC-F & CCACCTCCCACCCAATTCAT/3`-HEX/ \\
\hline 30- inputA & CTATCAАTCATCTTCAAАACAАAАССТCA \\
\hline 30- Clip1 & GGAGGAGGAGGAACACACTAAACTCAAAGATGATTGATAG \\
\hline 30- Clip8 & GGAGGAGGAGGACACACAACATTCACAAGATGATTGATAG \\
\hline 30- c-inputA & TGAGGTTTTGTTTTGAAGATGATTGATAG \\
\hline 30- c-Clip1 & CTATCAATCATCTTTGAGTTTAGTGTGTTCCTCCTCCTCC \\
\hline 30- c-Clip8 & CTATCAATCATCTTGTGAATGTTGTGTGTCCTCCTCCTCC \\
\hline 30- inputA' & СТССТCAATCTACC CAAAACAAAACCTCA \\
\hline 30-Clip2 & GGAGGAGGAGGAACTTCAACCCATACGGTAGATTGAGGAG \\
\hline 30- c-inputA' & TGAGGTTTTGTTTTGGGTAGATTGAGGAG \\
\hline 30-c-Clip2 & CTCCTCAATCTACCGTATGGGTTGAAGTTCCTCCTCCTCC \\
\hline 30-inputB & CCATCTTACACCTACATTATACCTCCATC \\
\hline 30-Clip3 & GGAGGAGGAGGACTCTACCACTTCTCTAGGTGTAAGATGG \\
\hline 30-Clip9 & GGTGGTGGTGGTCTAAACTACTCCCTTAGGTGTAAGATGG \\
\hline 30-c-inputB & GATGGAGGTATAATGTAGGTGTAAGATGG \\
\hline 30-c-Clip3 & ССATCTTACACCTAGAGAAGTGGTAGAGTCCTCCTCCTCC \\
\hline 30-c-Clip9 & CCATCTTACACCTAAGGGAGTAGTTTAGACCACCACCACC \\
\hline
\end{tabular}




\begin{tabular}{|c|c|}
\hline 30-inputB' & ATCTCСТАСТАТСТCATTATAССТССАТC \\
\hline 30-Clip4 & GGAGGAGGAGGATATCACTTTCACACAGATAGTAGGAGAT \\
\hline 30-c-inputB' & GATGGAGGTATAATGAGATAGTAGGAGAT \\
\hline 30-c-Clip4 & ATCTCCTACTATCTGTGTGAAAGTGATATCCTCCTCCTCC \\
\hline 30-AND1-T & $\begin{array}{l}\text { GGATGTTGATGGAGGTATAATGGTGTGAAAGTGATATGAGGTTTTGTTTTGT } \\
\text { GAGTTTAGTGTGT }\end{array}$ \\
\hline 30-AND1-M & CAAAACAAAACCTCATATCACT \\
\hline 30-AND1-O & 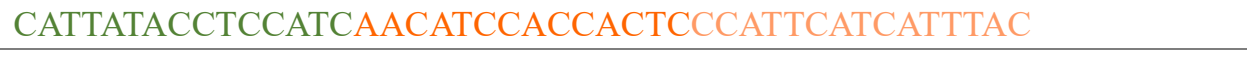 \\
\hline 30-AND2-T & $\begin{array}{l}\text { GGTGAAAGATGGAGGTATAATGGAGAAGTGGTAGAGTGAGGTTTTGTTTT } \\
\text { GGTATGGGTTGAAGT }\end{array}$ \\
\hline 30-AND2-M & CAAAACAAAACCTCACТCТАCC \\
\hline 30-AND2-O & САТТАТАССТССАТСТТTСАССТСАТССАССАТТСАТСАТTТАС \\
\hline 30-AND3-T & $\begin{array}{l}\text { AGGAAAAGATGGAGGTATAATGAGGGAGTAGTTTAGTGAGGTTTTGTTTTG } \\
\text { GTGAATGTTGTGTG }\end{array}$ \\
\hline 30-AND3-M & CAAAACAAAACCTCACTAAACT \\
\hline 30-AND3-O & САТТАТАССТССАТСТТТТССТАТСТАССССАССТСССАСССАА \\
\hline 30-OR-T & TGAACTTGTAAATGATGAATGGGTAAATAGTAAATG \\
\hline 30-OR-O & CCATGCAGCATTGACATCAGCAGGACGATCTGACGA \\
\hline 30-Clip5 & GGAGGAGGAGGACATTTACTATTTACGAGTGGTGGATGTT \\
\hline 30-Clip6 & GGAGGAGGAGGACATTTACTATTTACTGGATGAGGTGAAA \\
\hline 30-Clip7 & GGAGGAGGAGGATATGAAGTACAGTGCGTTGAGTGAACT \\
\hline 30-Clip10 & GGAGGAGGAGGA CACACAATCTCTACGGTAGATAGGAAAA \\
\hline 30-c-Clip5 & AACATCCACCACTCGTAAATAGTAAATGTCCTCCTCCTCC \\
\hline 30-c-Clip6 & TTTCACCTCATCCAGTAAATAGTAAATGTCCTCCTCCTCC \\
\hline 30-c-Clip 7 & AAGTTCACTCAACGCACTGTACTTCATATCCTCCTCCTCC \\
\hline 30-c-Clip10 & TTTTCCTATCTACCGTAGAGATTGTGTGTCCTCCTCCTCC \\
\hline
\end{tabular}

Full adder

\begin{tabular}{|c|c|}
\hline 31- ReporterS-Q & /5 BHQ1/ATGAATTGGGTGGGAGGTGGTGAGGTGTGGAGAGGA \\
\hline 31- ReporterS-F & CCACCTCCCACCCAATTCAT/3`-HEX/ \\
\hline 31- ReporterC-Q & /5 BHQ1/ATAGGATAAGGAAGAGGGTTTGAGTGATGTTGATGTG \\
\hline 31- ReporterC-F & AAACCCTCTTCCTTATCCTAT/36-FAM/ \\
\hline 31-inputA & TCCТАТСААТСАТСТTAААСССТСТТССТТА \\
\hline 31-inputA' & CATTCCACACCTTTAAAAACСCТСТTСCТTA \\
\hline 31-inputB & АТСТССТСТТССАСССАААСССТСТTССТТА \\
\hline 31-inputB' & ACATCTCCTACTATCTAAACCCTCTTCCTTA \\
\hline 31-inputC & CATTCAАТСССТTCССАААСССТСТТССТТА \\
\hline 31-inputC' & CTAACTCCACATACACAAАСССТСТТССТТА \\
\hline 31-c-inputA & TAAGGAAGAGGGTTTAAGATGATTGATAGGA \\
\hline 31-c-inputA' & TAAGGAAGAGGGTTTTTAAAGGTGTGGAATG \\
\hline 31-c-inputB & TAAGGAAGAGGGTTTGGGTGGAAGAGGAGAT \\
\hline 31-c-inputB' & TAAGGAAGAGGGTTTAGATAGTAGGAGATGT \\
\hline 31-c-inputC & TAAGGAAGAGGGTTTGGGAAGGGATTGAATG \\
\hline 31-c-inputC & TAAGGAAGAGGGTTTGTGTATGTGGAGTTAG \\
\hline \multirow[t]{2}{*}{ 31-AND1-T } & GTGGTGATAAGGAAGAGGGTTTTGAGAAAGGTGGTGTAAGGAAGAGGGT \\
\hline & TTTGTGTTGAAGAGTGTG \\
\hline 31-AND1-M & AАACССТСТTССТТАСАССACC \\
\hline
\end{tabular}




\begin{tabular}{|c|c|}
\hline 31-AND1-O & 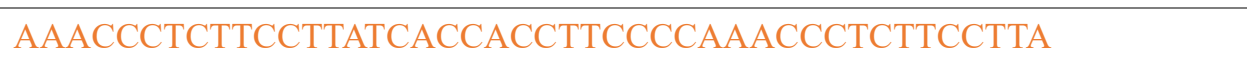 \\
\hline \multirow[t]{2}{*}{ 31-AND2-T } & AAGTAGTTAAGGAAGAGGGTTTGTTGGTAGTAGGATTAAGGAAGAGGGTT \\
\hline & TTTGAGGAGGTTTGGTA \\
\hline 31-AND2-M & AAACССТCTTCCTTAATCCTAC \\
\hline 31-AND2-O & AAACCCTCTTCCTTAACTACTTTCCACCTAAAACCCTCTTCCTTA \\
\hline \multirow[t]{2}{*}{ 31-AND3-T } & GTGGAAGTAAGGAAGAGGGTTTTGGAATGAGTGGTATAAGGAAGAGGGTT \\
\hline & TGGGTAGGATGAAGTGG \\
\hline 31-AND3-M & AААСССТСТТССТТАТАССАСТ \\
\hline 31-AND3-O & AААСССТСТТССТТАСТТССАСТААСССААААСССТСТТССТТА \\
\hline \multirow[t]{2}{*}{ 31-AND4-T } & GAGTTAGTAAGGAAGAGGGTTTGATGAGTGATGGTGTAAGGAAGAGGGTT \\
\hline & TGGAGTTGGTAGATGGA \\
\hline 31-AND4-M & АААСССТСТТССТТАСАССАТС \\
\hline 31-AND4-O & AАACССТСТТССТТАСТААСТСТАССТСТАААСССТСТТССТТА \\
\hline \multirow[t]{2}{*}{ 31-AND5-T } & GGTTGAATAAGGAAGAGGGTTTGAGTTGGTGAGGATTAAGGAAGAGGGTT \\
\hline & TTGATGGTAGATGGTGT \\
\hline 31-AND5-M & AАAСССТСТTССТTAАТССТCA \\
\hline 31-AND5-O & AAACCCTCTTCCTTATTCAACCACCTCTACCACCTCCCACCCAA \\
\hline \multirow[t]{2}{*}{ 31-AND6-T } & GGAGTAGTAAGGAAGAGGGTTTGTGATGGTGGTTGATAAGGAAGAGGGTT \\
\hline & TGAGTTGAGGGTAGGAT \\
\hline 31-ANT6-M & АААСССТСТТССТТАТСААССА \\
\hline 31-AND6-O & AАAСССТСТТССТТАСТАСТССТСССАССССАССТСССАСССАA \\
\hline \multirow[t]{2}{*}{ 31-AND7-T } & GAGGAGTTAAGGAAGAGGGTTTAGTGTTTGGTGTGGTAAGGAAGAGGGTT \\
\hline & TAGAGAAGTTGTGAGAG \\
\hline 31-AND7-M & АААСССТСТТССТТАССАСАСС \\
\hline 31-AND7-O & AАACССТСТTССТТААСТССТСТАТСТАСАААСССТСТТССТТА \\
\hline 31-Clip1 & GGAGGAGGAGGACACACTCTTCAACACAAAGATGATTGATAGGA \\
\hline 31- Clip2 & GGAGGAGGAGGACACCACCTTTCTCAAGATAGTAGGAGATGT \\
\hline 31- Clip3 & GAGAGAGAGTACCAAACCTCCTCAATTAAAGGTGTGGAATG \\
\hline 31- Clip4 & GAGAGAGAGATCCTACTACCAACGGGTGGAAGAGGAGAT \\
\hline 31- Clip5 & GAGAGAGAGCCACTTCATCCTACCCAAGATGATTGATAGGA \\
\hline 31- Clip6 & GAGAGAGAGTACCACTCATTCCAGGGTGGAAGAGGAGAT \\
\hline 31-Clip7 & GAGAGAGAGTCCATCTACCAACTCCTTAAAGGTGTGGAATG \\
\hline 31-Clip8 & GAGAGAGAGCACCATCACTCATCAGATAGTAGGAGATGT \\
\hline 31- Clip9 & AGAAGGGAGCTCTCACAACTTCTCTGGGGAAGGTGGTGA \\
\hline 31- Clip10 & AGAAGGGAGCTCTCACAACTTCTCTAGGTGGAAAGTAGT \\
\hline 31- Clip11 & GAGAGAGAGTCAACCACCATCACGTGTATGTGGAGTTAG \\
\hline 31- Clip12 & GAGAGAGAGACACCATCTACCATCATGGGTTAGTGGAAG \\
\hline 31- Clip13 & GAGAGAGAGACACCATCTACCATCAAGAGGTAGAGTTAG \\
\hline 31- Clip14 & GAAGGAAGAGCCACACCAAACACTGGAAGGTAGTAGTAGG \\
\hline 31- Clip15 & GAGAGAGAATCCTACCCTCAACTCGATGTATGTGGTGA \\
\hline 31-Clip16 & GAGAGAGAATCCTACCCTCAACTCGTGGATGAAGTAGT \\
\hline 31-Clip17 & GGAGGAGGAGGACCACACCAAACACTGGGAAGGGATTGAATG \\
\hline 31-Clip18 & GAGAAGAAGAAGCACACAATCTCTACTAGAGGTGGTTGAA \\
\hline 31-Clip19 & GAGAAGAAGAAGCACACAATCTCTACGGTAGGTGGAGTAG \\
\hline 31-Clip20 & GAGAGAGAGCACATCAACATCACTCTGGGTTAGTGGAAG \\
\hline 31-Clip21 & GGAGGAGGAGGACACATCAACATCACTCGTAGATAGAGGAGT \\
\hline 31-c-Clip1 & TCCTATCAATCATCTTTGTGTTGAAGAGTGTGTCCTCCTCCTCC \\
\hline
\end{tabular}




\begin{tabular}{ll}
\hline 31- c-Clip2 & ACATCTCCTACTATCTTGAGAAAGGTGGTGTCCTCCTCCTCC \\
\hline 31- c-Clip3 & CATTCCACACCTTTAATTGAGGAGGTTTGGTATCTCTCTC \\
\hline 31- c-Clip4 & ATCTCCTCTACACCCGTTGGAGAGGATCTCTCTCTC \\
\hline 31- c-Clip5 & TCCTATCAATCATCTTGGGTAGGATGAAGTGGCTCTCTCTC \\
\hline 31- c-Clip6 & ATCTCCTCTTCCACCCTGGAATGAGTGGTACTCTCTCTC \\
\hline 31- c-Clip7 & CATTCCACACCTTTAAGGAGTTGGTAGATGGACTCTCTCTC \\
\hline 31- c-Clip8 & ACATCTCCTACTATCTGATGAGTGATGGTGCTCTCTCTC \\
\hline 31- c-Clip9 & TCACCACCTTCCCCAGAGAAGTTGTGAGAGCTCCCTTCT \\
\hline 31- c-Clip10 & ACTACTTTCCACCTAGAGAAGTTGTGAGAGCTCCCTTCT \\
\hline 31- c-Clip11 & CTAACTCCACATACACGTGATGGTGGTTGACTCTCTCTC \\
\hline 31- c-Clip12 & CTTCCACTAACCCATGATGGTAGATGGTGTCTCTCTCTC \\
\hline 31- c-Clip13 & CTAACTCTACCTCTTGATGGTAGATGGTGTCTCTCTCTC \\
\hline 31- c-Clip14 & CCTACTACTACCTTCCGAGTTGAGGGTAGGATCTCTCTCTC \\
\hline 31- c-Clip15 & TCACCACATACATCGAGTTGAGGGTAGGATTCTCTCTC \\
\hline 31-c-Clip16 & ACTACTTCATCCACGAGTTGAGGGTAGGATTCTCTCTC \\
\hline 31-c-Clip17 & CATTCAATCCCTTCCCAGTGTTTGGTGGGTCCTCCTCCTCC \\
\hline 31-c-Clip18 & TTCAACCACCTCTAGTAGAGATTGTGTGCTTCTTCTTCTC \\
\hline 31-c-Clip19 & CTACTCCACCTACCGTAGAGATTGTGTGCTTCTTCTTCTC \\
\hline 31-c-Clip20 & CTTCCACTAACCCAGAGTGATGTTGATGTGCTCTCTCTC \\
\hline 31-c-Clip21 & ACTCCTCTATCTACGAGTGATGTTGATGTGTCCTCCTCCTCC \\
\hline
\end{tabular}

